\title{
Une introduction à la physique de la turbulence
}

\author{
An introduction to the physics of turbulence
}

\author{
J. C. Lelièvre
}

Université Pierre-et-Marie-Curie (Paris $6^{\circ}$ )

Institut de mécanique théorique et appliquée

\section{Introduction}

\subsection{Ecoulement turbulent}

En juin 1975, au colloque de la Société Française de Physique "Hydrodynamique physique et instabilités", Craya (1976) commençait sa revue de la turbulence par ces mots : "parler de turbulence est difficile. Essayer d'être clair et significatif en peu de temps devient acrobatique".

Il serait présomptueux de donner une définition précise de la turbulence, car chacun pourrait trouver une situation que la contredirait.

Comme Tennekes et Lumley (1972) ainsi que Favre et al. (1976), donnons les caractéristiques certaines, indéniables et essentielles d'un écoulement qualifié de turbulent :

- irrégularité. Le premier caractère est l'aspect irrégulier, désordonné et imprévisible ou aléatoire de l'écoulement ;

- diffusivité. C'est l'expérience fondamentale de Reynolds qui montre que les particules composant les filets colorés subissent un brassage intense et diffusent dans tout le tube. Il y a donc, par rapport à l'écoulement laminaire, un accroissement important des transferts de masse, de chaleur et de quantité de mouvement ;

- rotationnel et tridimensionnel. Le rotationnel de la vitesse présente de fortes fluctuations aléatoires;

- dissipation. Les écoulements turbulents sont essentiellement dissipatifs.

L'énergie cinétique d'agitation est dissipée en chaleur dans le fluide. Pour entretenir le mouvement, un rapport continu d'énergie est nécessaire.

- non linéarité. Entre les diverses échelles de fluctuations, il y a des interactions qui correspondent à un phénomène de transfert d'énergie dû au terme non linéaire de l'équation du mouvement.

On trouve dans le livre Favre et al. (1976, p. 5) des exemple d'écoulements turbulents en apparence, mais qui ne présentent pas l'ensemble des caractères mentionnés ci-dessus.

La turbulence se produit lorsque le nombre de Reynolds de l'écoulement est grand et est issue des instabilités de l'écoulement laminaire [Martin (1976)].

Landau (1944) propose un schéma physique élaboré à partir de $n$ transitions, chacune d'elles imposant au fluide d'osciller à une fréquence déterminée à partir de $n$ phases arbitraires. Le nombre $n$ augmentant avec le nombre de Reynolds, l'apparition de la turbulence serait graduelle et continuc.

Il ne semble pas que ce schéma soit confirmé par certaines expériences sur la transition, notamment celle de Gollub et Swinney (1975), qui conduisent à penser à une transition soudaine, comme l'indiquent Ruelle et Takens (1971) dans leur théorie sur la nature de la turbulence ${ }^{(1)}$.

La turbulence est une propriété des écoulements et non du fluide lui-même. Elle s'étudie, pour les fluides visqueux newtoniens incompressibles, avec l'équation de Navier :

$$
\frac{\partial \vec{q}}{\partial t}+(\vec{q} \cdot \vec{\nabla}) \vec{q}=-\frac{1}{p} \vec{\nabla} p+\nu \nabla^{2} \vec{q}
$$

où $p$ et $\vec{q}$ représentent respectivement la pression et la vitesse.

Les propriétés moléculaires du fluide n'interviennent que par la viscosité cinématique $\nu$.

(1) voir aussi l'article de Berge et Pommeau (1980). 
La description du mouvement se fera dans le cadre d'une théorie de milieu continu. Et, bien qu'il existe des fluctuations de très petites dimensions, la plus petite échelle rencontrée dans l'écoulement sera toujours beaucoup plus grande que le libre parcours moyen des molécules. On associe aussi à (1) l'équation de continuité :

$$
\vec{\nabla} \cdot \vec{q}=0
$$

\subsection{Méthodes d'étude de la turbulence}

\section{Historique}

La recherche d'une théorie satisfaisante de la turbulence a été marquée par des étapes remarquables : - étude de l'écoulement moyen [Boussinesq, Reynolds] sans recherche de la manière dont il dépend de la structure à petite échelle ;

- étude du champ à l'aide de la statistique appliquée [Taylor, Karman]. Cette étape s'est heurtée au problème de la fermeture du système des équations statistiques inférieures en nombre à celui des inconnues [Leslie (1976)].

- étude au moyen de la statistique mathématique [Kampe de Feriet, Kraichman].

\section{Aperçu sur les théories}

- Les théories heuristiques tendent de décrire la turbulence en termes de concepts : longueur de mélange de Prandtl, viscosité turbulente d'Heisenberg.

Parmi celles-ci les plus connues sont celles de Heisenberg (1948), Chandrasekhar (1949), Proudman (1951). Dans ces théories, le transfert de l'émergie turbulente des grandes échelles vers les petites est étudié au moyen de la viscosité turbulente.

D'autres hypothèses et approximations sur le transfert conduisent à d'autres formulations [Kovasznay (1948), Karman (1948), Goldstein (1951), Oboukhov (1954), Pao (1965), Leith (1967)] et donnent des expressions explicites pour le spectre d'énergie. Pour une comparaison critique, voir Ellison (1962).

- Les théories phénoménologiques. Parallèlement aux théories précédentes, issues des idées de Taylor, se sont développées des théories qui se déduisent d'hypothèses bien définies sur la nature de la turbulence : Homogénéité et isotropie du champ [Robertson (1940), Obukhov (1947)].

La turbulence ne dépend que de quelques grandeurs indépendantes, d'où l'utilisation de l'analyse dimensionnelle :

- les propriétés asymptotiques des écoulements sont indépendantes de la viscosité lorque le nombre de Reynolds tend vers l'infini;

- la structure locale, homogène et isotrope, est indépendante du mouvement à grande échelle et présente un caractère d'universalité [Kolmogorov (1941), Obukhov (1941), Weizacker (1948), Onsager (1945, 1949)].

- Les théorie probabilistes dont le but est de prédire le champ aléatoire en tout point de l'espace et à chaque instant moyennant un nombre restreint d'hypothèses [Van Atta (1972), Rose et Sulem (1978)].

\subsection{Aspect physique de la turbulence - intermittence}

Auprès des développements théoriques, des questions sur la physique de la turbulence se sont posées, notamment sur la dissipation de l'énergie turbulente aux petites échelles du mouvement.

C'est Landau ${ }^{(2)}$ qui a été amené à mettre en doute le caractère universel de la théorie de Kolmogorov en signalant la nature aléatoire de l'énergie dissipée. Pour prendre en compte la remarque de Landau, Kolmogorov (1962), Obukhov (1962) raffinent la théorie originale en introduisant une loi de probabilité spéciale, la loi lognormale, qui traduit le caractère intermittent des fluctuations de grand nombre d'ondes, phénomène constaté expérimentalement par Batchelor et Townsend (1949).

Novikov $(1963,1969,1976)$ a donné consistance à la nouvelle théorie en justifiant la loi log-normale par des considérations de similitude d'échelles.

Physiquement, l'intermittence correspond à une structure spatiale et temporelle qui montre une alternance de petites échelles d'intense activité et de larges domaines de repos. Cette intermittence étant d'autant plus prononcée que l'échelle concernée est petite.

La structure spatiale correspondante est certainement très compliquée et l'on aimerait pouvoir la visualiser au cours du temps par un moyen qui reste à découvrir.

Divers modèles de l'intermittence ont été élaborés : modèle géométrique de Frisch et al. (1974), modèle de cascade de Bell et Nelkin (1977), modèle fractal de Maudelbrot (1974), analogie avec les phénomènes critiques de Nelkin (1974).

\subsection{Bibliographie}

Dans cet article, nous développerons d'abord la théorie spectrale qui constitue une approche conventionnelle de la turbulence, mais qui demeure une bonne introduction pour la compréhension des phénomènes.

On trouvera dans des ouvrages plus denses les compléments de démonstrations esquissées dans le texte. Voir, par exemple, les livres de Hinze (1959), Batchelor (1970), Panchev (1971), Tennekes et Lumley (1972), Monin et Yaglom (1975), Favre et al. (1976), Frost et Moulden (1977), ainsi que de bons articles de revue; Chandrasekhar (1957), Lin (1959), Corrsin (1957, 1961), Lin et Reid (1963), Leslie (1973), Craya (1976), Rose et Sulem (1978).

D'excellents cours ont été dispensés ces dernières années, parmi ceux-ci notons Nihoul (1970), Lee (1970), Orszag (1973), Nelkin (1976) dont on s'est inspiré pour rédiger ce papier.

\section{Théorie spectrale de la turbulence homogène}

\subsection{Introduction et notations}

On considère, en chaque point de l'espace et à tout instant, les variables aléatoires qui caractérisent l'état du

(2) voir Landau et Lifchitz (1971, p. 158). 
fluide. Les interactions entre celles-ci rendent compte de la structure de la turbulence.

Pour étudier ces interactions, un moyen consiste à considérer les corrélations de la vitesse en deux ou plusieurs points de l'espace-temps.

Nous ne considèrerons que la turbulence homogène pour laquelle les valeurs moyennes de toute fonction des vitesses ou d'autres grandeurs turbulentes et de leurs dérivées sont invariantes pour toute translation arbitraire.

Un cas particulier est la turbulence isotrope qui est, de plus, invariante par rotation.

On appelle $\vec{U}$ la valeur moyenne et $\vec{u}$ la partie fluctuante de la vitesse locale $\vec{q}$ de sorte que :

$$
\vec{U}=\langle\vec{q}\rangle\langle\vec{u}\rangle=\overrightarrow{0}
$$

où $\langle\ldots\rangle$ représente l'opération de moyenne d'ensemble.

Dans un repère cartésien $\left(x_{1}, x_{2}, x_{3}\right)$, et lorsque l'on suppose $\vec{U}$ porté par l'axe $\vec{x}_{1}$, les équations 1 (1) et 1 (2) deviennent

$$
\begin{gathered}
\frac{\partial u_{i}}{\partial t}+U \frac{\partial u_{i}}{\partial x_{1}}+u_{j} \frac{\partial u_{i}}{\partial x_{j}}=-\frac{1 \partial p}{\rho \partial x_{i}}+v \frac{\partial^{2} u_{i}}{\partial x_{j} \partial x_{j}} \\
\frac{\partial u_{i}}{\partial x_{i}}=0
\end{gathered}
$$

On utilise, sauf spécification contraire, la convention de sommation sur l'indice répété, ainsi que la formule générale sur la moyenne d'un produit de dérivées pour un champ aléatoire $\vec{u}(\vec{x})$ [Batchelor (1970) p. 19].

\subsection{Notions fondamentales}

Tenseur des corrélations doubles - Energie cinétique d'agitation turbulente

Considérons, à l'instant $t$, le tenseur des corrélations doubles de la vitesse notée $\Gamma_{i j}$ défini par:

$$
\Gamma_{i j}(\vec{x}, \vec{r}, t)=\left\langle u_{i}(\vec{x}, t) u_{j}(\vec{x}+\vec{r}, t)\right\rangle
$$

Compte tenu de l'hypothèse d'homogénéité, la corrélation (4) sera écrite $\Gamma_{i j} \overrightarrow{(r)}$ et l'on aura la symćtrie :

$$
\Gamma_{i j}(\vec{r})=\Gamma_{j i}(-\vec{r})
$$

de plus, d'après (3), on voit que :

$$
\frac{\partial}{\partial r_{j}} \Gamma_{i j}(\vec{r})=0
$$

Pour $\vec{r}=\overrightarrow{0}$, la trace de $\Gamma_{i j}$ représente, au facteur $1 / 2$ près, l'énergie cinétique moyenne d'agitation turbulente au point et à l'instant considéré, pour l'unité de masse de fluide, on la note:

$$
\frac{1}{2}<u^{2}>=\frac{1}{2}\left[\Gamma_{11}(0)+\Gamma_{22}(0)+\Gamma_{33}(0)\right]
$$

Tenseur spectral d'énergie - Spectre de l'énergie cinétique

Considérons la transformée de Fourier de $\Gamma_{i j}(\vec{r})$, soit :

$$
E_{i j}(\vec{K})=\frac{1}{(2 \pi)^{3}} \int \Gamma_{i j}(\vec{r}) e^{-\overrightarrow{i K} \cdot \vec{r}} d \vec{r}
$$

$E_{i j}(\vec{K})$ est appelé tenseur spectral d'énergie qui présente certaines propriétés de parité que l'on peut découvrir.

La condition d'incompressibilité (6) entraîne, pour $E_{i j}(\vec{K})$, la relation :

$$
K_{i} E_{i j}(\vec{K})=K_{j} E_{j i}(\vec{K})=0
$$

La fonction $E_{u u}(\vec{K})$ définie par :

$$
E_{u u}(\vec{K})=\frac{1}{2}\left[E_{11}(\vec{K})+E_{22}(\vec{K})+E_{33}(\vec{K})\right]
$$

est appelée densité spectrale 3-dim. de l'énergie cinétique d'agitation, on a en effet:

$$
\int E_{u u}(\vec{K}) d \vec{K}=\frac{1}{2}<u^{2}>
$$

On définit aussi le spectre d'énergie $E_{u}(K)$ en prenant l'intégrale de $E_{u u}(\vec{K})$ sur une sphère de rayon $|\vec{K}|$ de sorte que :

$$
\frac{1}{2}<u^{2}>=\int_{0}^{\infty} E_{u}(K) d K
$$

où $E_{u}(K)$ est une fonction non négative.

Vecteur flux d'énergie - Fonction transfert d'énergie Considérons le vecteur:

$$
\begin{aligned}
N_{j}=\frac{1}{2}<u_{i}(\vec{x}) & u_{i}(\vec{x}+\vec{r}) u_{j}(\vec{x})- \\
& -u_{i}(\vec{x}) u_{i}(\vec{x}+\vec{r}) u_{j}(\vec{x}+\vec{r})>
\end{aligned}
$$

qui a la dimension d'un flux d'énergie par unité de masse de fluide. Notons $W(\vec{K})$ la transformée de Fourier de $\left(\partial N_{j} / \partial r_{j}\right)$ :

$$
W(K)=\frac{1}{(2 \pi)^{3}} \int \frac{\delta N_{j}}{\delta r_{j}} e^{-i \vec{K} \cdot \vec{r}} d \vec{r}
$$

et $T(\vec{K})$ la fonction obtenue en intégrant $W(\vec{K})$ sur la sphère de rayon $|\vec{K}|$, soit :

$$
\int_{0}^{\infty} T(K) d K=\int W(\vec{K}) d \vec{K}
$$

Il vient, d'après $(14)$ :

$$
\begin{gathered}
\int_{0}^{\infty} T(\vec{K}) d \vec{K}=\frac{1}{(2 \pi)^{3}} \int d \vec{K} \int \frac{\partial N_{j}}{\partial r_{j}} e^{-\vec{i} \vec{K} \circ \vec{r}} d \vec{r}= \\
\left.\quad=\int \frac{\partial N_{j}}{\delta r_{j}} \delta \vec{r}\right) d \vec{r}=\left.\frac{\partial N_{j}}{\partial r_{j}}\right|_{\vec{r}=\overrightarrow{0}}
\end{gathered}
$$

où $\delta \overrightarrow{(r)}$ est la fonction de Dirac. 
Soit, compte tenu de la définition (13) et de la condition (3) :

$$
\left.\frac{\partial N_{j}}{\partial r_{j}}\right|_{\substack{r=0 \\ \text { a }}}=-\frac{\partial}{\partial x_{j}}\left(u_{i} u_{i} u_{j}\right)=0
$$

d'où la relation, utile pour la suite :

$$
\int_{0}^{\infty} T(K) d K=\int W(\vec{K}) d \vec{K}=0
$$

\subsection{Forme du spectre d'énergie pour les petits nombres d'ondes}

Considérons un mouvement généré par une distribution homogène et stationnaire de forces impulsives $\vec{g}(\vec{x})$ aléatoires, de moyenne nulle [Saffman (1967)].

Il s'agit, par exemple, du mouvement initial pris par un fluide contenu dans une enceinte que l'on secoue brusquement. Les échelles comparables à la taille du récipient, c'est-à-dire les plus petits nombres d'onde, sont d'abord concernées. La viscosité étant sans effet au début du mouvement, les équations sont:

$$
\begin{gathered}
\vec{u}=\vec{\nabla} p+\vec{g} \\
\vec{\nabla} \cdot \vec{u}=0
\end{gathered}
$$

où $p$ est la pression impulsive.

Appelons $\vec{U}(\vec{K}), \quad P(\vec{K}), \vec{G}(\vec{K})$ les transformées de Fourier respectives de $\vec{u}, \vec{p}, \vec{g}$, il vient :

$$
\begin{gathered}
\vec{U}=i \vec{K} p+\vec{G} \\
\vec{K} \cdot \vec{U}=0
\end{gathered}
$$

En multipliant (21) par $\vec{K}$ et compte tenu de (22) on a :

$$
U_{i}=\left(\delta_{i j}-\frac{K_{i} K_{j}}{K^{2}}\right) G_{j}(\vec{K})
$$

soit, d'après (4) et (8) :

$$
E_{i j}(\vec{K})=\left(\delta_{i k}-\frac{K_{i} K_{k}}{K^{2}}\right)\left(\delta_{j l}-\frac{K_{i} K_{l}}{K^{2}}\right) \phi_{k l}(\vec{K})
$$

$\underset{\mathrm{où}}{\rightarrow} \phi_{k I}(\vec{K})$ est le tenseur spectral associé à la distribution $\vec{g}(\vec{x})$.

Saffman (1967), en faisant des hypothèses sur les propriétés statistiques du système de forces $\vec{g}(\vec{x})$, montre que les spectre d'énergie $E_{u}(K)$ obtenu d'après (24) doit prendre la forme :

$$
E_{u}(K)=A K^{2}+O\left(K^{4}\right)
$$

où $A$ est une constante positive, en général non nulle.

Lorsque $A$ est égal à zéro, Batchelor (1970, p. 37) indique que la forme prise par $E_{u}(K)$ doit être :

$$
E_{u}(K)=B K^{4}+O\left(K^{6}\right)
$$

Le spectre d'énergie cinétique tombe donc rapidement à zéro, au voisinage des petits nombres d'onde. Cette remarque prend de l'intérêt lorsque l'on veut opérer une troncature auprès de $K=0$.

\subsection{Turbulence atténuée}

Lorsque la turbulence est faible, c'est le cas au bout d'un temps assez long dans l'expérience du paragraphe précédent, le terme non linéaire $(\vec{u} \cdot \vec{\nabla}) \vec{u}$ devient négligeable devant $\vec{u} / \partial t$, il en est de même des termes de pression devant les termes de viscosité. Les équations du mouvement se réduisent à :

$$
\begin{aligned}
& \frac{\partial \vec{u}}{\partial t}=\nu \nabla^{2} \vec{u} \\
& \vec{\nabla} \cdot \vec{u}=0
\end{aligned}
$$

de sorte que le spectre d'énergie vérifie :

$$
\frac{\partial}{\partial t} E(K, t)=-2 \nu K^{2} E(K, t)
$$

dont la solution générale est :

$$
E(K, t)=C(K) e^{-2 \nu K^{2} t}
$$

La forme de $C(K)$ peut être trouvée en faisant tendre $K$ vers zéro, ce qui nous reporte au paragraphe précédent. Si (25) s'applique, Saffman (1967) conclut que la décroissance de l'énergie cinétique doit être en $t^{-3 / 2}$. Si, au contraire, c'est (26) qui est vraie, alors l'énergie turbulente décroit, dans la phase finale, comme $t^{-5 / 2}$ [Batchelor (1970, p. 72)]. C'est ce qui a été vérifié par Batchelor et Townsend (1949) derrière une grille de turbulence.

D'après (12) on a :

$$
\frac{1}{2}<u^{2}>=\int_{0}^{\infty} C(K) e^{-2 \nu K^{2} t} d K
$$

qui indique que l'énergie cinétique est essentiellement contenue dans les fluctuations de nombres d'ondes voisins de zéro, ceci étant d'autant plus vrai que $t$ est grand.

\subsection{Dynamique de la turbulence développée}

Nous étudions maintenant le cas où l'équation de Navier complète (2) s'applique.

Pour simplifier l'écriture, on utilise la notation suivante :

$$
u_{i}(\vec{x}+\vec{r}, t)=u_{i}^{\prime}(\vec{x}, t)=u_{i}^{\prime}
$$

Multiplions (2) par $u_{i}^{\prime}$, il vient :

$$
\begin{aligned}
u_{i}^{\prime} \frac{\partial u_{i}}{\partial t}+U u_{i}^{\prime} \frac{\partial u_{i}}{\partial x_{1}}+u_{i}^{\prime} u_{j} \frac{\partial u_{i}}{\partial x_{j}} & = \\
& -\frac{1}{\rho} u_{i}^{\prime} \frac{\partial p}{\partial x_{i}}+v u_{i}^{\prime} \frac{\delta^{2} u_{i}}{\partial x_{j} \partial x_{j}}
\end{aligned}
$$

On obtient une équation analogue à (33) en permutant $\vec{x}$ et $\vec{x}^{\prime}$ que l'on additionne à (33), ce qui donne :

$$
\begin{aligned}
& \frac{\partial}{\partial t}\left(u_{i} u_{i}^{\prime}\right)+U\left(u_{i}^{\prime} \frac{\partial u_{i}}{\partial x_{1}}+u_{i} \frac{\partial u_{i}^{\prime}}{\partial x_{1}^{\prime}}\right)+u_{i}^{\prime} u_{j} \frac{\partial u_{i}}{\partial x_{j}}+u_{i} u_{j}^{\prime} \frac{\partial u_{i}^{\prime}}{\partial x_{j}^{\prime}} \\
& =-\frac{1}{\rho}\left(u_{i}^{\prime} \frac{\partial p}{\partial x_{i}}+u_{i} \frac{\partial p^{\prime}}{\partial x_{i}^{\prime}}\right)+\nu\left(u_{i}^{\prime} \frac{\partial^{2} u_{i}}{\partial x_{j} \partial x_{j}}+u_{i} \frac{\partial^{2} u_{i}^{\prime}}{\partial x_{j}^{\prime} \partial x_{j}^{\prime}}\right)(
\end{aligned}
$$


Comme $\vec{u}_{i}$ est indépendant: de $\vec{x}^{\prime}$ et que $\vec{u}_{i}^{\prime}$ est indépendant de $\vec{x}$, il vient, compte tenu de (3):

$$
\begin{gathered}
\frac{\partial}{\partial t}\left(u_{i} u_{i}^{\prime}\right)+U\left[\frac{\partial}{\partial x_{1}}\left(u_{i} u_{i}^{\prime}\right)+\frac{\partial}{\partial x_{1}^{\prime}}\left(u_{i} u_{i}^{\prime}\right)\right]+ \\
+\frac{\partial}{\partial x_{j}}\left(u_{i} u_{j}^{\prime} u_{j}\right)+\frac{\partial}{\partial x_{j}^{\prime}}\left(u_{i} u_{i}^{\prime} u_{j}^{\prime}\right)= \\
-\frac{1}{\rho}\left[\frac{\partial}{\partial x_{i}}\left(u_{i}^{\prime} p\right)+\frac{\partial}{\partial x_{i}^{\prime}}\left(u_{i} p^{\prime}\right)\right]+\nu\left[\frac{\delta^{2}\left(u_{i} u_{i}^{\prime}\right)}{\partial x_{j} \partial x_{j}}+\frac{\delta^{2}\left(u_{i} u_{i}^{\prime}\right)}{\partial x_{j}^{\prime} \partial x_{j}^{\prime}}\right]
\end{gathered}
$$

On prend maintenant la moyenne d'ensemble de (35) et l'on applique les règles de dérivation [Batchelor (1970) p. 19]. On utilise aussi l'équation de continuité (2) et le fait que $p$ est indépendant de $\vec{x}^{\prime}$, alors (35) conduit au résultat simple :

$$
\frac{\partial}{\partial t}<u_{i} u_{i}^{\prime}>-2 \frac{\partial N_{j}}{\partial r_{j}}=2 \nu \frac{\delta^{2}}{\partial r_{j} \partial r_{j}}<u_{i} u_{i}^{\prime}>
$$

où $N_{j}$ est le vecteur flux d'énergie défini en (13).

Considérons le tenseur des corrélations doubles envisagé en (4) et notons $2 \Gamma \overrightarrow{(\vec{r})}$ la trace :

$$
2 \Gamma(\vec{r})=\Gamma_{11}(\vec{r})+\Gamma_{22} \overrightarrow{(r)}+\Gamma_{33}(\vec{r})
$$

il vient pour (36) :

$$
\frac{\partial \Gamma}{\partial t}=2 v \nabla^{2} \Gamma+\vec{\nabla} \cdot \vec{N}
$$

où les opérateurs $\nabla^{2}$ et $\vec{\nabla}$ doivent être considérés dans l'espace $\vec{r}$.

Effectuons, sur (38), l'opération de transformée de Fourier et utilisons les définitions (8), (10) et (14), on a :

$$
\frac{\partial}{\partial t} E_{u u}(\vec{K}, t)=2 \nu K^{2} E_{u u}(\vec{K}, t)+W(\vec{K}, t)
$$

En intégrant les termes de (39) sur une sphère de rayon $|\vec{K}|$, on obtient :

$$
\frac{\partial}{\partial t} E_{u}(K, t)=2 \nu K^{2} E_{u}(K, t)+T(K, t)
$$

où l'on rappelle que $E_{u}(K)$ est le spectre d'énergie et $T(K)$ la fonction de transfert (15).

L'équation (40) donne le taux de décroissance de $E_{u}(K)$ à condition de connaître $T(K)$. La recherche

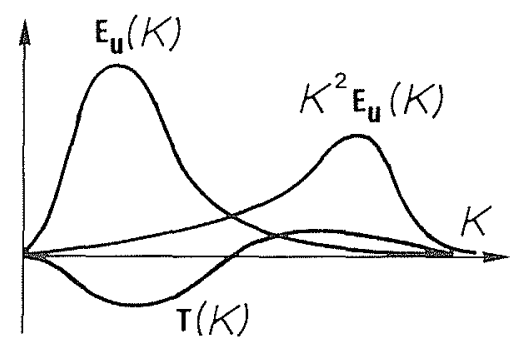

Figure 1 - Disposition relative des spectres de la turbulence. dë cette fonction, a priori inconnue, constitue un problème central de la théorie de la turbulence. Les variations comparées des termes de $(40)$ sont indiquées sur la figure 1 . Le spectre $K^{2} E_{u}(K)$, appelé spectre de dissipation, opère essentiellement dans le domaine des grands nombres d'ondes.

\subsection{Conservation de l'énergie}

D'après (12), le taux de décroissance de l'énergie cinétique est :

$$
\frac{\partial}{\partial t}\left(\frac{1}{2}<u^{2}>\right)=\frac{\partial}{\partial t} \int_{0}^{\infty} E_{u}(K, t) d K
$$

Notons, pour la suite, $\left\langle\epsilon_{u}\right\rangle$, la quantité suivante :

$$
<\epsilon_{u}>=2 v \int_{0}^{\infty} K^{2} E_{u}(K, t) d K
$$

Intégrons les termes de (40) sur toutes les valeurs de $K$. il vient, d'après la propriété (18) et en utilisant (41) et (42) :

$$
\left.\frac{\partial}{\partial t}\left(\frac{1}{2}<u^{2}\right\rangle\right)=-\left\langle\epsilon_{u}>\right.
$$

Le terme $T(K)$, qui provient du terme non linéaire de l'équation de Navier, n'apporte aucune contribution au bilan d'énergie (43). Il sert au transfert de l'énergie cinétique, d'où l'appellation fonction de transfert, parmi les nombres d'ondes de l'espace de Fourier.

Les questions que l'on se pose portent sur le domaine de nombres d'ondes concemé, sur le sens et le mécanisme du transfert.

On sait, on en reparlera plus loin, qu'il existe un domaine, qualifié d'inertiel, dans lequel $T(K)$ opère le transfert de l'énergie contenue dans les grandes échelles (petits nombres d'ondes) vers les petites échelles (grands nombres d'ondes).

Dans l'espace physique, les gros tourbillons alimentés en énergie cinétique par l'extérieur, se divisent par étirage $^{(3)}$ en plus petits tourbillons jusqu'à cc que leur taille soit suffisamment faible pour que la viscosité devienne influente. L'énergie cinétique est alors dissipée en chaleur dans le fluide, le tourbillon s'atténue puis s'annihile.

\subsection{Dissipation de l'énergie turbulente}

La puissance spécifique dissipée dans un fluide classique par les frottements visqueux s'écrit [Batchelor (1967, p. 151)] :

$$
\phi=2 \nu\left(e_{i j} e_{i j}-\frac{1}{3} e_{l l}^{2}\right)=2 \nu\left(e_{i j}-\frac{1}{3} e_{l l} \delta_{i j}\right)^{2}
$$

où $e_{i j}$ est le tenseur qui caractérise la déformation du champ turbulent :

$$
e_{i j}=\frac{1}{2}\left(\frac{\partial u_{i}}{\partial x_{j}}+\frac{\partial u_{j}}{\partial x_{i}}\right)
$$

(3) Vortex stretching. 
On considère le taux moyen de dissipation en opérant la moyenne de (44), ce qui donne, compte tenu de (3):

$$
\left\langle\phi>=2 \nu<e_{i j} e_{i j}>=\frac{\nu}{2}<\left(\frac{\partial u_{i}}{\partial x_{j}}+\frac{\partial u_{j}}{\partial x_{i}}\right)^{2}>\right.
$$

ou encore :

$$
\langle\phi\rangle=\nu\left[\left\langle\left(\frac{\partial u_{i}}{\partial x_{j}}\right)^{2}>+<\frac{\partial u_{i}}{\partial x_{j}} \frac{\partial u_{j}}{\partial x_{i}}>\right]\right.
$$

Soit, en utilisant les formules sur la moyenne des dérivées [Batchelor (1970) p. 19] (47) devient :

$$
\langle\phi\rangle=v\left[\left\langle\left(\frac{\partial u_{i}}{\partial x_{j}}\right)^{2}>+\frac{\partial^{2}}{\partial x_{i} \partial x_{j}}<u_{i} u_{j}\right\rangle\right]
$$

En turbulence homogène, où (6) s'applique, le deuxième terme du crochet est nul, de sorte que :

$$
<\phi>=\nu<\left(\frac{\partial u_{i}}{\partial x_{j}}\right)^{2}>=\nu<\frac{\partial u_{i}}{\partial x_{j}} \frac{\partial u_{i}}{\partial x_{j}}>
$$

On calcule (49) en utilisant, à nouveau, les formules sur la moyenne des dérivées et en procédant par passage à la limite comme suit :

$$
<\phi>=\lim _{r \rightarrow 0} v<\frac{\partial u_{i}}{\partial x_{j}} \frac{\partial u_{i}^{\prime}}{\partial x_{j}^{\prime}}>
$$

d'où :

$$
<\phi>=-2 \nu \frac{\partial^{2} \Gamma(0)}{\partial r_{j}^{2}}
$$

où $\Gamma(\vec{r})$ est donné par $(37)$.

D'après (8), en prenant la transformée de Fourier inverse, on a :

$$
\Gamma_{i j}(\vec{r})=\int E_{i j}(\vec{K}) e^{i \vec{K} \cdot \vec{r}} d \vec{K}
$$

soit en calculant (51), d'après (37), (52) et (10), il vient :

$$
<\phi>=2 \nu \int_{0}^{\infty} K^{2} E_{u}(K) d K
$$

En comparant avec (42), on voit que :

$$
\left\langle\epsilon_{u}>=\langle\phi\rangle\right.
$$

Autrement dit, l'égalité (43) qui traduit la loi de décroissance de l'énergie cinétique indique que cette dernière est entièrement dissipée dans le fluide.

Il est usuel de conserver la notation $\left\langle\epsilon_{u}\right\rangle$ pour le taux de dissipation de l'énergie cinétique turbulente, ce que nous ferons désormais.

\section{Echelles de la turbulence}

\subsection{Echelles énergétiques et échelles dissipatives}

A l'aide de la quantité $\left\langle u^{2}\right\rangle^{1 / 2}$ représentant la vitesse quadratique moyenne d'agitation turbulente et du taux moyen de dissipation $\left\langle\epsilon_{u}\right\rangle$, on peut former par l'analyse dimensionnelle, les échelles de longueur $L$ et de temps $T$ et, par suite, de vitesse $V$ en écrivant :
$\left.\left.L=\left\langle u^{2}\right\rangle^{3 / 2}<\epsilon_{u}\right\rangle^{-1} T=\left\langle u^{2}\right\rangle<\epsilon_{u}\right\rangle^{-1}$

$$
V=\left\langle u^{2}>^{1 / 2}\right.
$$

Ces échelles, constituées sans prendre en compte la viscosité cinématique $v$, sont appelées échelles énergétiques ou échelles externes de la turbulence.

Si nous considérons maintenant le couple $\nu$ et $\left\langle\epsilon_{u}\right\rangle$, les échelles constituées sont:

$$
\begin{aligned}
\eta=\nu^{3 / 4}<\epsilon_{u}>^{-1 / 4} t_{\eta}=\nu^{1 / 2}<\epsilon_{u} & >^{-1 / 2} \\
v_{\eta} & =\nu^{1 / 4}<\epsilon_{u}>^{1 / 4}
\end{aligned}
$$

Ces échelles sont appelées échelles dissipatives ou échelles internes de la turbulence, ou encore échelles de Kolmogorov.

On définit le nombre de Reynolds $R_{L}$ de la turbulence par :

$$
\left.R_{L}=\frac{\left\langle u^{2}\right\rangle^{1 / 2} L}{v}=\left\langle u^{2}\right\rangle^{2} \nu^{-1}<\epsilon_{u}\right\rangle^{-1}
$$

Si l'on forme le nombre de Reynolds $R_{\eta}$ à l'aide des échelles dissipatives, on voit que :

$$
R_{\eta}=\frac{v_{\eta} \eta}{v}
$$

A l'aide de (3) et (1), on exprime les échelles dissipatives sous la forme:

$$
\eta=\frac{L}{R_{L}^{3 / 4}} \quad t_{\eta}=\frac{T}{R_{L}^{1 / 2}} \quad v_{\eta}=\frac{V}{R_{L}^{1 / 4}}
$$

On sait que la turbulence développée correspond à un nombre de Reynolds $R_{L}$ très grand, de sorte que l'on a les relations d'ordre de grandeur.

$$
\eta \ll L \quad t_{\eta} \ll T \quad v_{\eta}<V
$$

\subsection{Echelle de Taylor}

Compte tenu de 2 (53), on introduit une longueur $\lambda$, liée à la dissipation, par l'expression :

$$
\frac{<u^{2}>^{1 / 2}}{2 \lambda^{2}}=\int_{0}^{\infty} K^{2} E_{u}(K) d K
$$

Cette nouvelle échelle, ou échelle de Taylor, sert souvent de référence dans les expériences sur la turbulence.

D'après (7), elle s'évalue à partir du spectre de dissipation et peut aussi se déterminer à partir de la courbure à l'origine de la fonction de corrélation, conformément à 2 (51).

En turbulence homogène, il existe deux échelles $\lambda_{f}$ et $\lambda_{g}$ respectivement appelées microéchelle longitudinale et latérale reliées entre elles, lorsque la turbulence est isotrope par:

$$
\lambda_{f}=\lambda_{g} \sqrt{2}
$$

On considère alors, dans les expériences, le nombre de Reynolds :

$$
R_{\lambda}=\frac{\left\langle u^{2}>^{1 / 2} \lambda_{g}\right.}{\nu}
$$


d'où les relations pratiques en turbulence isotrope [Hinze (1959, p. 185)] :

$$
\begin{aligned}
R_{L} & =\frac{1}{15} R_{\lambda}^{2} \\
\frac{\left\langle u^{2}\right\rangle^{1 / 2}}{v_{\eta}} & =\frac{1}{(15)^{1 / 4}} R_{\lambda}^{1 / 2} \\
\frac{\lambda_{g}}{\eta} & =(15)^{1 / 4} R_{\lambda}^{1 / 2} \\
\frac{L}{\lambda_{g}} & =\frac{1}{15} R_{\lambda} \\
\frac{L}{\eta} & =\frac{1}{(15)^{3 / 4}} R_{\lambda}^{3 / 2}
\end{aligned}
$$

Notons que $\lambda_{g}$, quantité mesurable, permet la détermination du taux de dissipation $\left\langle\epsilon_{u}\right\rangle$ par la relation:

$$
<\epsilon_{u}>=15 \nu \frac{\left.<u^{2}\right\rangle}{\lambda_{g}^{2}}
$$

avec $\left\langle u^{2}\right\rangle=3 \sigma_{u}^{2}$ où $\sigma_{u}^{2}$ est la variance d'une composante de $\vec{u}$.

\section{Domaine du transfert de l'énergie}

On a vu [2(18)] que l'intégrale de la fonction transfert d'énergie est nulle. De ce fait, on conclut qu'elle ne contribue pas au bilan de la conservation de l'énergie 2 (43).

Reprenons 2 (18):

$$
\int_{0}^{\infty} T(K) d K=0
$$

que nous écrivons sous la forme équivalente :

$$
\int_{K}^{\infty} T(K) d K=-\int_{0}^{K} T(K) d K=\pi(K)
$$

$\pi(K)$ représente le flux d'énergie pénétrant dans la sphère de rayon $|K|$ dans l'espace de Fourier.

Utilisons l'argument de Craya (1975) qui suppose l'existence d'un domaine de l'espace de Fourier composé de grands nombres d'ondes et appelé domaine d'équilibre, dans lequel la variation $\partial E_{u} / \partial t$ intervenant dans 2 (40) est négligeable. C'est-à-dire :

$$
\pi(K)=2 \nu \int_{0}^{\infty} K^{2} E_{u}(K) d K
$$

On suppose, de plus, qu'il existe à l'origine du domaine précédent un domaine, qualifié d'inertiel, tel que la dissipation y soit négligeable. De sorte que (3) s'écrit :

$$
\pi(K)=2 \nu \int_{0}^{\infty} K^{2} E_{u}(K) d K=\text { cte }=\left\langle\epsilon_{\mathrm{u}}>\right.
$$

où l'on a introduit $\left\langle\epsilon_{u}\right\rangle$ conformément à 2 (53) et 2 (54). (4) donne une deuxième signification à $\left\langle\epsilon_{u}\right\rangle$. C'est le flux d'énergie qui pénètre dans le domaine inertiel et le traverse sans modification.

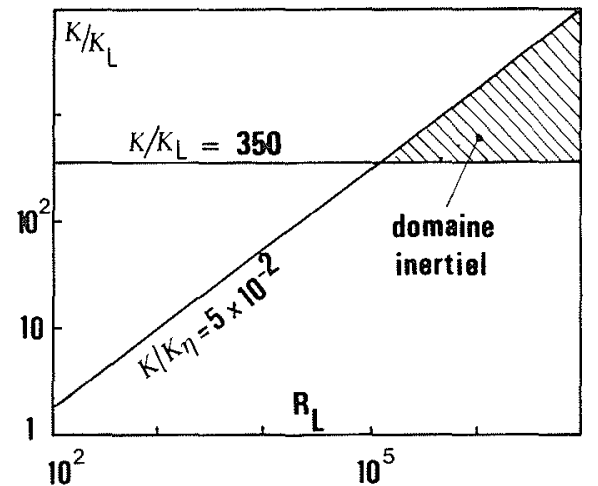

Figure 2 - Conditions d'existence du domaine inertiel.

Compte tenu de la définition des échelles ( $\$ 3$ ) le domaine inertiel est constitué des nombres d'onde $K$ tels que :

$$
K_{L} \sim \frac{1}{L} \ll K \ll K_{\eta} \sim \frac{1}{\eta}
$$

Tennekes et Lumley (1973, p. 266) précisent les conditions pour lesquelles les domaines précédents existent.

Lorsque le nombre de Reynolds de la turbulence $R_{L}$ est supérieur à $10^{2}$, on peut raisonnablement s'attendre à un domaine d'équilibre. Mais il faut que $R_{L}$ soit supérieur à $10^{5}$ pour qu'il y ait une possibilité de zone inertielle, mais cette condition n'est pas suffisante. Dans le diagramme de la figure 2, la condition (5), compte tenu de la première égalité 3 (5), indique un exemple de domaine permis. Corrsin (1962), en se fondant sur l'échelle de Taylor, admet l'existence d'un domaine inertiel dans le spectre d'énergie de la turbulence si l'on vérifie :

$$
R_{\lambda}>500
$$

On peut se rendre compte à l'aide de 3 (10) que (6) ne serait pas suffisant puisque moins contraignant que le critère de Tennekes et Lumley.

\section{Phénoménologie de la turbulence}

\subsection{Cascade d'énergie}

Pour se faire une idée physique de la turbulence, on imagine le champ turbulent composé de cellules en interactions. Ces cellules ou tourbillons (eddies) sont autosemblables et rien ne permet de les distinguer, fors leur taille. Ils sont supposés former un ensemble statistique en équilibre. L'espace de Fourier est divisé en couches sphériques d'épaisseur $\Delta K$ dont les rayons sont en progression géométrique. On considère les rayons successifs $K_{n-1}, K_{n}, K_{n+1}$ et l'on note :

$$
\Delta K_{n}=K_{n+1}-K_{n-1}
$$

Soit $a$ la raison de la progression. Alors :

$$
\Delta K_{n}=K_{n}\left(a-\frac{1}{a}\right)
$$


Il est judicieux, comme l'on fait Tennekes et Lumley (1973, p. 258), de choisir $a$ de sorte que (2) devienne simplement :

$$
\Delta K_{n}=K_{n}
$$

Ces auteurs en déduisent une interprétation énergétique du tourbillon : un tourbillon est une perturbation dont l'énergie $E_{u}(K)$ est contenue dans la bande $\Delta K$ autour de $K$. Le spectre correspondant aurait l'allure représentée sur la figure 3. Dans l'espace physique, la fonction de corrélation présente une oscillation de longueur d'onde égale à $K^{-1}$ et amortie sur la distance $(\Delta K)^{-1}$. On en déduit que la taille du tourbillon est

$$
l=(\Delta K)^{-1}=K^{-1}
$$

Le nombre $n$ de cellules en interaction serait [Craya $(1975)]$ :

$$
n=\frac{3}{8} \frac{1}{\operatorname{Ln} a} \operatorname{Ln} R_{L}
$$

Tennekes (1977) précise le modèle en disant que le tourbillon de taille initiale $l_{n-1}$ donne naissance à $a^{2}$ tourbillons de dimension $l_{n}$.

Le mécanisme détaillé de l'interaction conduisant à cette division, c'est-à-dire à ce transfert d'énergie, n'est pas encore clair mais on comprend le modèle dénommé cascade d'énergie.

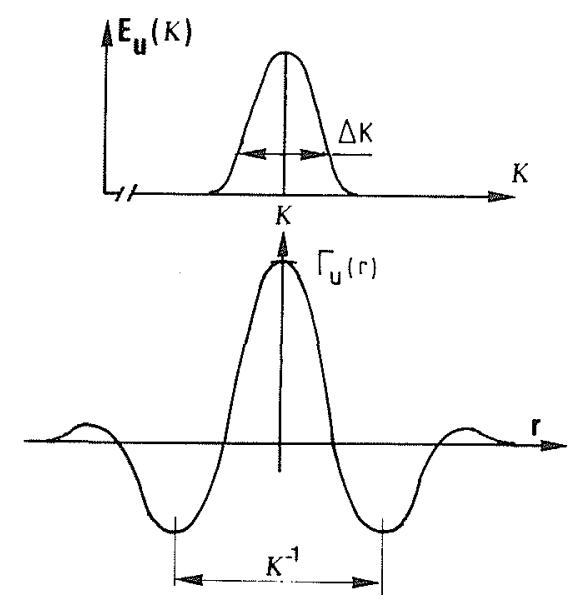

Figure 3 - Interprétation énergétique du tourbillon.

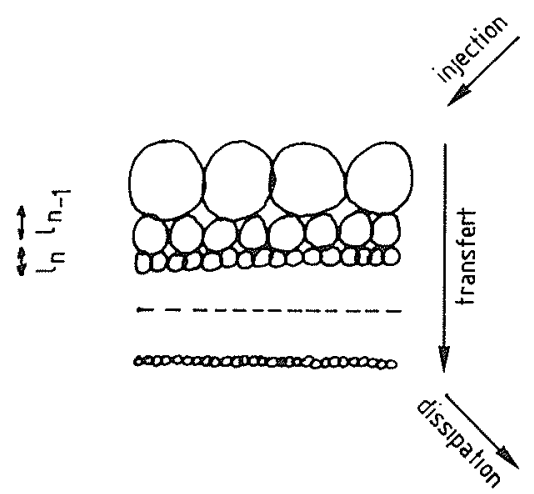

Figure 4 - Modèle de cascade (sans in termittence).
A la manière de Rose et Sulem (1978), on visualise la cascade schématiquement sur la figure 4 . Le mouvement aux grandes échelles est entretenu par une injection continue d'énergie qui est transférée dans les échelles plus petites puis dissipée en chaleur dans le fluide. Le passage de l'énergie s'accompagne de la division successive des tourbillons.

Reprenons le propos de Craya (1975) "La mécanique de la turbulence n'est pas celle de l'équipartition de l'énergie entre un grand nombre de degrés de liberté dans un système conservatif, mais celle d'un flux d'énergie entre une source et un puits dans un système dissipatif".

Si l'on note $E_{n}$ l'énergie cinétique spécifique du tourbillon $K_{n}$ on a :

$$
E_{n}=\int_{K_{n}}^{K_{n+1}} E_{u}(K) d K=E_{u}\left(K_{n}\right) \Delta K_{n}
$$

en supposant un spectre en puissance pour $E_{u}(K)$. Ce qui se vérifiera a posteriori.

A l'aide de $E_{n}$ et $K_{n}$ on introduit les échelles correspondant à hiérarchie $K_{n}$ par :

$$
\begin{gathered}
l_{n}=K_{n}^{-1} \\
v_{n}=E_{n}^{1 / 2}=\left[E_{u}\left(K_{n}\right)\right]^{1 / 2} K_{n}^{1 / 2} \\
t_{n}=\frac{l_{n}}{v_{n}}=E_{n}^{1 / 2} K_{n^{\prime \prime}}^{-1}=\left[E_{u}\left(K_{n}\right)\right]^{1 / 2} K_{n}^{-3 / 2}
\end{gathered}
$$

Le temps $t_{n}$ est le temps de distorsion qui correspond au temps nécessaire à la division du tourbillon de taille $l_{n}$.

$v_{n}$ est la variation de la vitesse sur l'espace occupé par le tourbillon suivi dans son mouvement d'advection par le tourbillon qui lui a donné naissance.

Le flux d'énergie $\pi\left(K_{n}\right)$ quittant la lame sphérique $\Delta K_{n}$ et pénétrant dans la lame suivante est:

$$
\pi\left(K_{n}\right)=\frac{E_{n}}{t_{n}}=\frac{v_{n}^{3}}{l_{n}}=\left[E_{u}\left(K_{n}\right)\right]^{3 / 2} K_{n}^{5 / 2}
$$

Dans le domaine inertiel, $\pi\left(K_{n}\right)$ est constant et égal à la dissipation $\left\langle\epsilon_{u}\right\rangle$. On a donc, d'après (9):

$$
E_{u}(K)=\left\langle\epsilon_{u}>^{2 / 3} K^{-5 / 3}\right.
$$

Le spectre obtenu, à une constante multiplicative près, de l'ordre de l'unité, est le spectre de Kolmogorov (1941) pour l'énergie cinétique. D'où, d'après $(5$, l'énergie $E_{n}$ :

$$
E_{n}=\left\langle\epsilon_{u}>^{2 / 3} K_{n}^{-2 / 3}\right.
$$

et, d'après (7) et (8) :

$$
\begin{aligned}
& v_{n}=\left\langle\epsilon_{u}\right\rangle^{1 / 3} K_{n}^{-1 / 3} \\
& t_{n}=\left\langle\epsilon_{u}\right\rangle^{-1 / 3} K_{n}^{-2 / 3}
\end{aligned}
$$

La dissipation $\left\langle\epsilon_{u}\right\rangle$ s'évalue, par exemple, à l'étape finale de la cascade. C'est-à-dire lorsque $K=K_{\eta}=\eta^{-1}$. Soit, d'après 3 (2) :

$$
<\epsilon_{u}>=\nu^{3} K_{\eta}^{4}
$$


Le temps de distorsion pour $K=K_{\eta}$ vaut donc, d'après (13) :

$$
t_{\eta}=\frac{1}{\nu K_{\eta}^{2}}
$$

Or, $\left(\nu K^{2}\right)^{-1}$ n'est autre que le temps d'amortissement, ou durée de vie, du tourbillon de nombre d'onde $K$ qui, d'après (15), devient égal au temps de distorsion.

Le phénomène de division ne saurait subsister puisque la viscosité annihile immédiatement le tourbillon créé. La cascade cesse dès que $K \sim K_{\eta}$ et l'énergie se dissipe dans le fluide.

\subsection{Intermittence}

Le modèle précédent est corrigé en tenant compte du fait que tout l'espace n'est pas nécessairement rempli par les tourbillons.

L'aspect correspondant à cette idée est schématisé sur la figure 5, qui est à comparer à la figure 4 .

Soit $n$ le nombre de tourbillons de dimension $l_{n}$ issus de la $(n-1)^{\text {èrne }}$ génération. On suppose que ces tourbillons occupent seulement une fraction $\beta_{n}$ de l'espace rempli par les tourbillons $l_{n-1}$.

Une hypothèse élémentaire sur le fractionnement est exprimée par la relation :

$$
\beta_{n}=\beta^{n}
$$

avec :

$$
0 \leqq \beta \leqq 1
$$

Rose et Sulem (1978) indiquent la correction à apporter aux expressions (11), (12) et (13) pour tenir compte de cette nouvelle idée.

$$
\begin{aligned}
& E_{n}=\left\langle\epsilon_{u}>^{2 / 3} K_{n}^{-2 / 3}\left(l_{0} K_{n}\right)^{-\mu}\right. \\
& v_{n}=<\epsilon_{u}>^{1 / 3} K_{n}^{-1 / 3}\left(l_{0} K_{n}\right)^{-\mu} \\
& t_{n}=\left\langle\epsilon_{u}>^{-1 / 3} K_{n}^{-2 / 3}\left(l_{0} K_{n}\right)^{-\mu}\right.
\end{aligned}
$$

où l'exposant $\mu$ peut s'exprimer en fonction de $\beta ; l_{0}$ étant la taille du tourbillon initial de la cascade. Le spectre d'énergie (10) est aussi modifié et devient :

$$
E_{u}(K)=\left\langle\epsilon_{u}>^{2 / 3} K^{-5 / 3}\left(l_{0} K\right)^{-\mu}\right.
$$

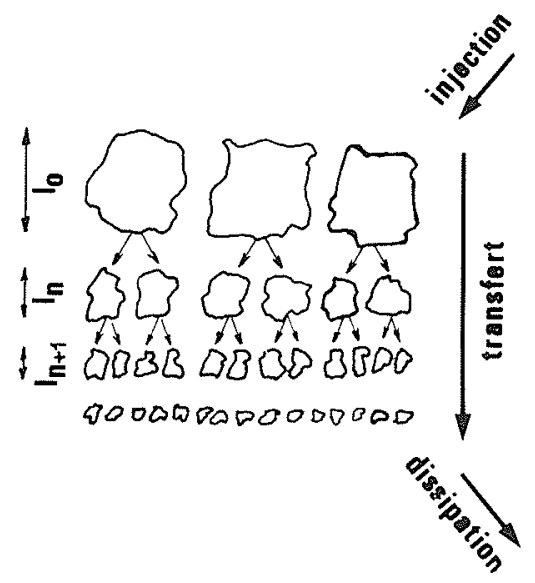

Figure 5 - Modèle de cascade (avec intermittence).
Les résultats modifiés ne sont donc plus indépendants des grandes échelles du mouvement.

La théorie évalue $\mu=0,05$, ce qui apporte une petite correction à la loi des $-5 / 3$. Encore faut-il que le nombre de Reynolds de la turbulence soit très élevé. $\mathrm{La}$ vérification ne peut s'effectuer que sur des écoulements géophysiques et non sur une expérience de laboratoire où-les nombres de Reynolds obtenus sont généralement trop faibles. Cette vérification exigerait, au demeurant, une précision difficilement accessible aux mesures actuelles en turbulence.

\section{Formulation originelle de Kolmogorov (1941)}

On admet que les échelles concernées ont un comportement isotrope. Ce n'est évidemment pas le cas, en général, des grandes structures de l'écoulement qui sont sensibles aux conditions aux frontières qui génèrent le mouvement.

Pour s'affranchir des grosses structures, on considère, au lieu de la vitesse locale, la différence des vitesses $\Delta \vec{u}$. Pour cela, on forme le tenseur dont les composantes sont les fonctions de structure $S_{i j} \overrightarrow{(r)}$ :

$$
S_{i j}(\vec{r})=\left\langle\left(u_{i}^{\prime}-u_{i}\right)\left(u_{i}^{\prime}-u_{j}\right)\right\rangle
$$

où $u_{i}$ sont les composantes de la vitesse au point $\vec{x}$ et $u_{i}^{\prime}$ les composantes de la vitesse au point $\vec{x}+\vec{r}$. Ce que l'on écrit encore :

$$
S_{i j} \overrightarrow{(r)}=\left\langle\Delta u_{i} \Delta u_{j}\right\rangle
$$

Lorsque $r$ n'est pas trop grand $(r \ll L), S_{i j}(\vec{r})$ ne dépend que de la structure locale supposée homogène et isotrope.

La forme générale de $S_{i j}(\vec{r})$ relève des résultats concernant les champs homogènes et isotropes. On introduit $\Delta u_{r}$ et $\Delta u_{t}$, projections de $\Delta \overrightarrow{\vec{u}}$ sur $\overrightarrow{\vec{r}}$ et sur le plan perpendiculaire à $\vec{r}$.

\subsection{Domaine de l'équilibre et domaine inertiel}

La première hypothèse de Kolmogorov (1941) consiste à supposer l'existence d'un domaine de $\vec{r}$ pour lequel les paramètres de similitude sont $v_{\eta}$ pour la différence de vitesse, et $\eta$ pour les distances. D'après $3(2)$ on a :

$$
v_{\eta}=\nu^{1 / 4}<\epsilon_{u}>^{1 / 4} \quad \eta=\nu^{3 / 4}<\epsilon_{u}>^{-1 / 4}
$$

Autrement dit, seules la viscosité $\nu$ et la dissipation $\left\langle\epsilon_{u}\right\rangle$ interviennent dans ce domaine appelé domaine de l'équilibre.

Les moments, d'ordre quelconque $p$, de la variable aléatoire $\Delta u_{r}$ ne peuvent s'écrire que sous la forme :

$$
<\Delta u_{r}^{p}>=v_{\eta}^{p} f_{p}\left(\frac{r}{\eta}\right)
$$

où $f_{p}$ est une fonction non dimensionnée universelle de $\frac{r}{\eta}$

$\mathrm{La}$ deuxième hypothèse de Kolmogorov suppose, à l'intérieur du domaine précédent, une zone, dite 
inertielle, dans laquelle la viscosité a un rôle négligeable. Cela impose au deuxième membre de (4) d'être indépendant de $\nu$ et, par suite, à la fonction $f_{p}$ d'être une fonction puissance :

$$
f_{p}\left(\frac{r}{\eta}\right)=A_{p}\left(\frac{r}{\eta}\right)^{p / 3}
$$

où $A_{p}$ est une constante de l'ordre de l'unité.

En particulier, les variances $\left\langle\Delta u_{r}^{2}\right\rangle$ et $\left\langle\Delta u_{t}^{2}\right\rangle$ s'écriront :

$$
\begin{aligned}
& <\Delta u_{r}^{2}>=A_{2}<\epsilon_{u}>^{2 / 3} r^{2 / 3} \\
& <\Delta u_{t}^{2}>=\frac{4}{3} A_{2}<\epsilon_{u}>^{2 / 3} r^{2 / 3}
\end{aligned}
$$

Ces résultats ont été trouvés, pour la première fois, par Kolmogorov (1941) et Obukhov (1941).

\subsection{Zone d'amortissement visqueux}

Pour les distances de corrélation $r$ très petites devant l'échelle dissipative $\eta$, on peut supposer [Landau et Lifchitz (1966, p. 157)] que :

$$
\Delta u_{r} \sim r
$$

de sorte que la variance correspondante aura un comportement de la forme :

$$
<\Delta u_{r}^{2}>=a_{2} r^{2}
$$

où $a_{2}$ est une constante qui s'évalue en considérant la dissipation pour un champ laminaire. Le résultat est :

$$
\begin{aligned}
& <\Delta u_{r}^{2}>=\frac{1}{15} \frac{\left\langle\epsilon_{u}>\right.}{\nu} r^{2} \\
& \left.<\Delta u_{t}^{2}\right\rangle=\frac{2}{15} \frac{\left\langle\epsilon_{u}>\right.}{\nu} r^{2}
\end{aligned}
$$

Pour les valeurs de $r$ de l'ordre de $L$, il faut considérer la structure à grande échelle et opérer le raccordement [Saffman (1967)] en tenant compte au besoin de l'anisotropie.

L'étude au voisinage de $r=\eta$ s'effectue en faisant des hypothèses sur la forme du spectre d'énergie $E_{u}(K)$ au voisinage de $K=K_{\eta}$ [Pao (1965)] et en calculant les

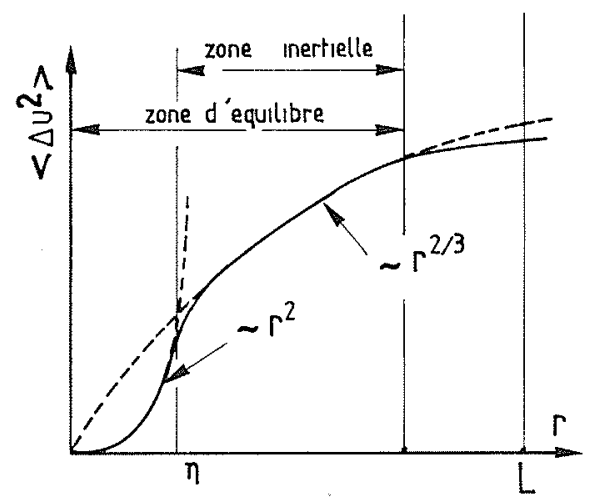

Figure 6 - Variance des fluctuations de la différence des vitesses en deux points en fonction de leur distance. fonctions de structure correspondantes par transformée de Fourier.

On montre que les fonctions de structure (6) et (7) donnent bien un spectre d'énergie en puissance $-5 / 3$, conformément à 5 (10).

La figure 6 représente l'allure des variations de la variance des fluctuations de la différence des vitesses en fonction de la distance entre les deux points considérés.

\section{Nouvelle formulation de Kolmogorov (1962)}

\subsection{Loi Log-normale}

On considère les moments d'ordre 3 et d'ordre 4 de $\Delta u_{r}$ que l'on écrit sous la forme non dimensionnée :

$$
D(r)=-\frac{\left\langle\Delta u_{r}^{3}\right\rangle}{\left\langle\Delta u_{r}^{2}\right\rangle^{3 / 2}} \quad A(r)=\frac{\left.<\Delta u_{r}^{4}\right\rangle}{\left\langle\Delta u_{r}^{2}\right\rangle^{2}}
$$

$D(r)$ et $A(r)$ sont respectivement appelés facteur de dissymétrie et facteur d'aplatissement de la distribution statistique des valeurs prises par $\Delta u_{r}$.

Lorsque les fluctuations de $\Delta u_{r}$ sont gaussiennes on trouve : $D=0$ et $A=3$, ce qui découle immédiatement des propriétés de la loi de Gauss. Ces valeurs sont bien trouvées expérimentalement lorsque les distances de corrélation $r$ sont très supérieures à l'échelle dissipative $\eta$, résultat provenant du fait que les fluctuations de $u_{r}$ sont elles-mêmes gaussiennes comme en témoigne bon nombre d'expériences (Fig. 7). Si l'on considére les facteurs de dissymétrie et d'aplatissement pour des valeurs de $r$ beaucoup plus petites que l'échelle fondamentale, c'est-à-dire les limites :

$$
D_{0}=\lim _{r \rightarrow 0} D(r) \quad A_{0}=\lim _{r \rightarrow 0} A(r)
$$

on constate expérimentalement que $D_{0}$ et $A_{0}$ s'écartent très largement des valeurs gaussiennes (respectivement 0 et 3 ).

A nombre de Reynolds de la turbulence modéré, $R_{\lambda} \sim 20$, Batchelor et Townsend (1949) trouvent :

$$
A_{0}=4 \quad D_{0}=-0,4
$$

D'autres résultats confirment l'écart par rapport à la loi de Gauss et indiquent aussi une croissance de $A_{0}$ et $D_{0}$ en fonction de $R_{\lambda}$.

Wyngaard et Pao (1972) rassemblent les résultats d'un grand nombre d'expériences couvrant la gamme $20 \leqq R_{\lambda} \leqq 1000$. La figure 8 . montre que $D_{0}$ varie à

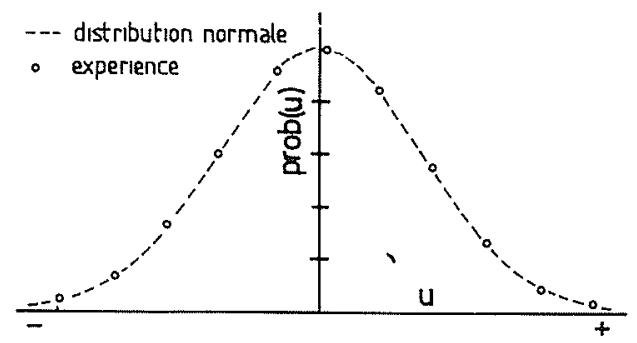

Figure 7 - Loi de probabilité des fluctuations de la vitesse, 


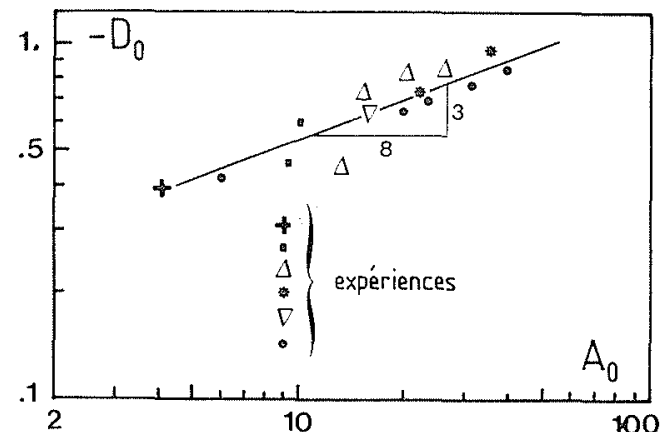

Figure 8 -Facteur de disymétrie en fonction du facteur d'aplatissement.

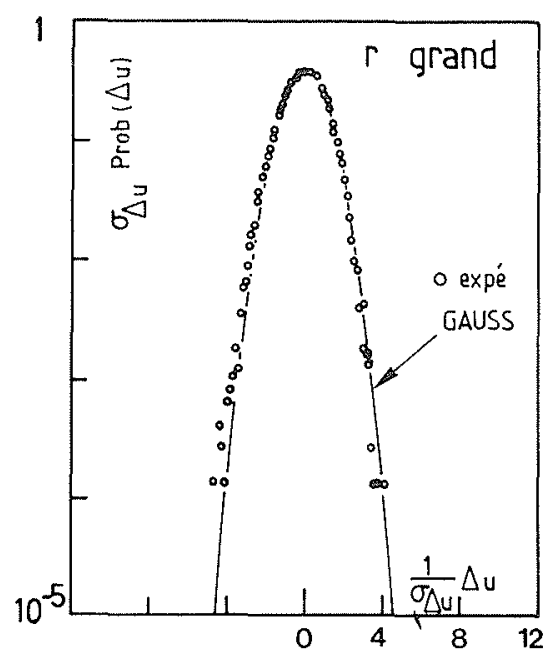

Figure 9 - Loi de probabilité des fluctuations de la différence des vitesses en deux points lorsque la distance de ceux-ci est comparable à l'échelle énergétique de laturbulence.

peu près comme la puissance $3 / 8$ du facteur d'aplatissement $A_{0}$. Penchons-nous maintenant sur la loi de probabilité de $\Delta u$. Lorsque $r$ est grand, on observe la loi de Gauss (Fig. 9). Mais lorsque l'écart $r$ devient comparable à l'échelle dissipative on constate une divergence notable par rapport au comportement gaussien (Fig. 10).

Cette divergence s'accentue lorsque le nombre de Reynolds de la turbulence croit, ainsi que lc montre la figure 11 de l'article de Wyngaard et Pao (1972). On voit que les grandes et les petites valeurs de $\Delta u$ ont une probabilité plus grande de se produire que dans le cas gaussien. Il y a donc des périodes de calme suivies de périodes d'activité intense, on dit que le processus aléatoire est intermittent.

Le fondement de la nouvelle théorie de Kolmogorov (1962) se trouve dans les constatations expérimentales rappelées ci-dessus et dans la remarque de Landau ${ }^{(4)}$. Celui-ci indique que la dissipation $\epsilon_{u}$ doit être traitée comme une grandeur qui fluctue beaucoup dans l'espace et dans le temps.

Il convient, comme Obukhov (1962), de considérer au lieu de la constante $\left\langle\epsilon_{u}\right\rangle$, une moyenne $\epsilon_{r}$ qui

(4) Landau et Lifchitz (1971, p. 158).

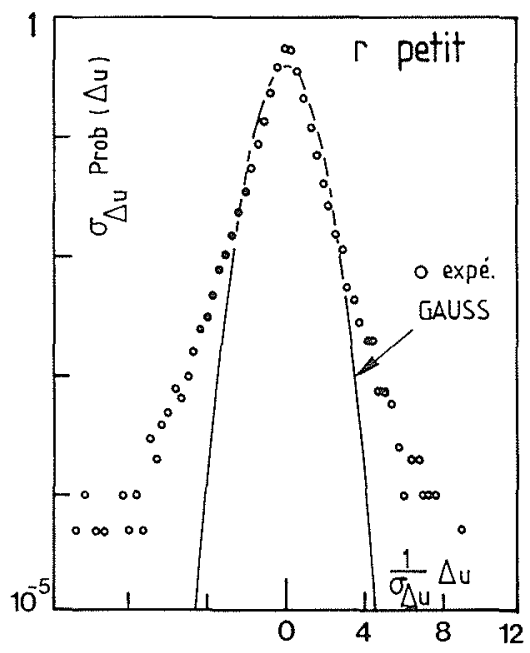

Figure 10 - Loi de probabilité des fluctuations de la différence des vitesses en deux points lorsque la distance de ceux-ci est comparable à l'échelle dissipative de la turbulence.

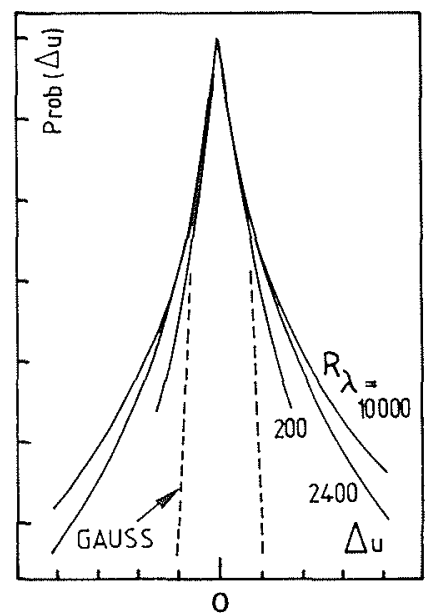

Figure 11 - Loi de probabilité des fluctuations de la différence des vitesses en deux poin ts pour différents nombres de Reynolds de la turbulence.

dépend de l'échelle considérée. Tel est le cas de la moyenne spatiale :

$$
\epsilon_{r}=\frac{1}{\frac{4}{3} \pi r^{3}} \int_{\left(\Sigma_{r}\right)} \epsilon_{u}(\vec{x}+\vec{r}) d r
$$

où $\left(\Sigma_{r}\right)$ est la sphère de rayon $r$ centrée en $\vec{x}^{(5)}$.

Comme $\epsilon_{u}$ est moyenné sur un volume fini, on a affaire à une quantité aléatoire de $\vec{x}$ et de $t$, mais dont la moyenne d'ensemble $\left\langle\epsilon_{r}>\right.$ est $\left\langle\epsilon_{u}\right\rangle$.

Partant de $\epsilon_{r}$, il est possible, comme au paragraphe 3.1., de constituer les échelles $v_{r}$ et $\eta_{r}$ :

$$
\eta_{r}=\nu^{3 / 4} \epsilon_{r}^{-1 / 4} \quad v_{r}=\nu^{1 / 4} \epsilon_{r}^{1 / 4}
$$

et de formuler les deux première hypothèses de Kolmogorov.

(5) pour une analyse précise de la théorie modifiée de Kolmogorov voir Gurvich et Yaglom (1967). 
Le comportement statistique de la différence des vitesses admet (5) comme paramètres de similitude, soit :

$$
<\Delta u_{r}^{p}>=<\left(r^{1 / 3} \epsilon_{r}^{1 / 3}\right)^{p}>f_{p}\left(\frac{r}{\eta_{r}}\right)
$$

S'il existe une zone inertielle, $\left\langle\Delta u_{r}^{p}\right\rangle$ doit être indépendant de la viscosité. En particulier pour la variance, on aura :

$$
\left.<\Delta u_{r}^{2}\right\rangle=B_{2}\left\langle\epsilon_{r}^{2 / 3}\right\rangle r^{2 / 3}
$$

expression à comparer à $6(6)$

Pour aller plus loin, il faut connaître la loi de probabilité de $\epsilon_{r}$ afin de calculer la moyenne qui intervient dans (7).

L'hypothèse nouvelle ou troisième hypothèse de Kolmogorov consiste à envisager une loi log-normale pour les fluctuations de $\epsilon_{r}$. Une telle loi par sa forme, traduit, avec une certaine fidélité, le comportement intermittent de l'activité turbulente.

Yaglom (1966) justifie ce choix en considérant une hiérarchie de tourbillons autosemblables indépendants. Voir aussi Gurvich et Yaglom (1967) pour les calculs détaillés.

La loi log-normale est certainement mieux adaptée qu'une loi de Gauss pour traduire les fluctuations de la dissipation [Orszag (1973)]. On se reportera aux articles de Novikov (1971), Kraichnan (1974) et Mandelbrot (1974b) qui étudient son champ d'application en cherchant les justifications théoriques.

\subsection{Corrections introduites par la nouvelle formulation}

Suivons Orszag (1973) ou Craya (1976) pour les résultats. On suppose que les fluctuations de $\log \epsilon_{r}$ sont distribuées suivant une loi de Gauss dont les paramètres, moyenne et variance, seront notés :

$$
\begin{gathered}
m_{r}=\left\langle\operatorname{Ln} \epsilon_{r}\right\rangle \\
D_{r}^{2}=\left\langle\left(\operatorname{Ln} \epsilon_{r}-m_{r}\right)^{2}\right\rangle
\end{gathered}
$$

La loi de probabilité $p\left(\epsilon_{r}\right)$ s'écrit sous la forme :

$$
p\left(\epsilon_{r}\right)=\frac{1}{\sqrt{2 \pi} D_{r} \epsilon_{r}} \exp \left[-\frac{\left(\operatorname{Ln} \epsilon_{r}-m_{r}\right)^{2}}{2 D_{r}^{2}}\right]
$$

Les paramètres $m_{r}$ et $D_{r}$ s'expriment en fonction de la dissipation moyenne $\left\langle\epsilon_{u}\right\rangle$ et de la taille $l_{0}$ du tourbillon générateur de la cascade $[\S 5.1$.$] :$

$$
\begin{aligned}
& D_{r}= \begin{cases}A+9 \mu \operatorname{Ln} \frac{l_{0}}{r} & r \gg \eta \\
A+9 \mu \operatorname{Ln} \frac{l_{0}}{\eta} & r \ll \eta\end{cases} \\
& m_{r}= \begin{cases}\operatorname{Ln}<\epsilon_{u}>-\frac{A}{2}-\frac{9 \mu}{2} \operatorname{Ln} \frac{l_{0}}{r} & r \gg \eta \\
\operatorname{Ln}<\epsilon_{u}>-\frac{A}{2}-\frac{9 \mu}{2} \operatorname{Ln} \frac{l_{0}}{\eta} & r \ll \eta\end{cases}
\end{aligned}
$$

où $A$ peut dépendre des conditions de génération de la turbulence, et $\mu$ est une constante universelle.

A partir de (10) on calcule les moments d'ordre quelconque $p$ de la variable aléatoire $\epsilon_{r}$, ce qui donne :

$$
<\epsilon_{r}^{p}>=\exp \left(p m_{r}+\frac{1}{2} p^{2} D_{r}^{2}\right)
$$

Soit, compte tenu de (11) et (13) ou (12) et (14) :

$$
<\epsilon_{r}^{p}>=\left\{\begin{array}{l}
\text { Cte }<\epsilon_{u}>^{p}\left(\frac{l_{0}}{r}\right)^{9 \mu \frac{p(p-1)}{2}} r \gg \eta \\
\text { Cte }<\epsilon_{u}>^{p}\left(\frac{l_{0}}{\eta}\right)^{9 \mu \frac{p(p-1)}{2}} \\
r \ll \eta
\end{array}\right.
$$

D'où la variance, dans le domaine inertiel :

$$
<\Delta u_{r}^{2}>=C_{2}<\epsilon_{u}>^{2 / 3} r^{2 / 3}\left(\frac{l_{0}}{r}\right)^{-\mu}
$$

On note que, dans le cas $r \ll \eta$ la variance 6 (10) reste inchangée.

Les facteurs d'aplatissement $A(r)$ et de dissymétrie $D(r)$ définis par (1) peuvent se calculer avec la nouvelle loi, ce qui donne :

$$
A(r)=\operatorname{Cte}\left(\frac{l_{0}}{r}\right)^{4 \mu} D(r)=\operatorname{Cte}\left(\frac{l_{0}}{r}\right)^{\frac{3 \mu}{2}}
$$

On en déduit

$$
D(r) \propto[A(r)]^{3 / 8}
$$

Ce résultat semble cohérent avec les résultats expérimentaux de la figure 8, ce qui étaye la théorie modifiée de Kolmogorov.

Quant au spectre d'énergie, il est modifié comme indiqué en 5 (20).

\section{Spectre d'énergie cinétique dans le domaine des grands nombres d'ondes.}

Au paragraphe 2.6., on a calculé le taux de dissipation $\left\langle\epsilon_{u}\right\rangle$ en fonction du spectre d'énergie cinétique $E_{u}(K)$. Soit :

$$
<\epsilon_{u}>=2 \nu \int_{0}^{\infty} K^{2} E_{u}(K) d K
$$

Lorsque l'on introduit dans (1) le spectre de Kolmogorov 5 (10) ou le spectre corrigé de l'intermittence 5 (21), on voit que l'intégrale (1) est divergente pour les grandes valeurs de $K$. La théorie de Kolmogorov n'est donc pas adaptée pour décrire la turbulence dans le domaine de l'amortissement visqueux.

Divers auteurs ont apporté leur contribution à l'étude de $E_{u}(K)$ pour les nombres d'ondes $K \gtrsim K_{\eta}$ : en particulier, les auteurs des théories heuristiques cités au paragraphe 1.2. et notamment Heisenberg (1948) et 
Tableau 1. - Spectre d'énergie $E_{u}(K)$ selon divers auteurs.

On a posé $\widetilde{K}=K / K_{\eta} \cdot \alpha_{H}, \beta_{H}, \alpha_{N}, \alpha_{P}$ et $\gamma$ sont des constantes.

\begin{tabular}{|c|c|c|}
\hline HEISENBERG (19.48) & $\left(\frac{8<\varepsilon_{g}}{9 \gamma}\right)^{2 / 3} K^{-5 / 3}\left[1+\frac{8 \gamma^{3}}{3 \gamma^{2}<\varepsilon_{\alpha}} K^{4}\right]^{-4 / 3}$ & BATCHELOR(1970, p.130) \\
\hline NOYIKOV (1961) & $\begin{array}{l}\alpha_{H}<\varepsilon_{k}^{2 / 3} K^{-5 / 3}\left[1+\beta_{H} \tilde{K}^{4}\right]^{-4 / 3} \\
\left.1,30<\varepsilon_{u}\right\rangle^{2 / 3} K^{-5 / 3} \exp \left(-1.76 \tilde{K}^{2}\right)\end{array}$ & $\begin{array}{l}\text { avec } \beta_{H}=\left(\frac{3 \alpha_{H}}{2}\right)^{3} \\
\text { TATARSKii }(4971, \text { p. } 57)\end{array}$ \\
\hline PAO (1965) & $\begin{array}{l}\alpha_{N}<\varepsilon_{Q}^{2 / 3} K^{-5 / 3} \exp -1.17 \alpha_{N} \tilde{K}^{2} \\
\alpha_{P}<\xi_{Q}^{2 / 3} K^{-5 / 3} \exp \left(-\frac{3}{2} \alpha_{P} \tilde{K}^{6 / 3}\right)\end{array}$ & \\
\hline $\begin{array}{l}\text { Ajustement sur les } \\
\text { experiences de } \\
\text { GRANT et al. (1962) }\end{array}$ & $(1,35 \pm 0,06)<\varepsilon_{2}^{2 / 3} K^{-5 / 3} \exp \left(-2,18 \tilde{K}^{1 / 2}\right)$ & TATARSKii $(1971$, p. 58) \\
\hline
\end{tabular}
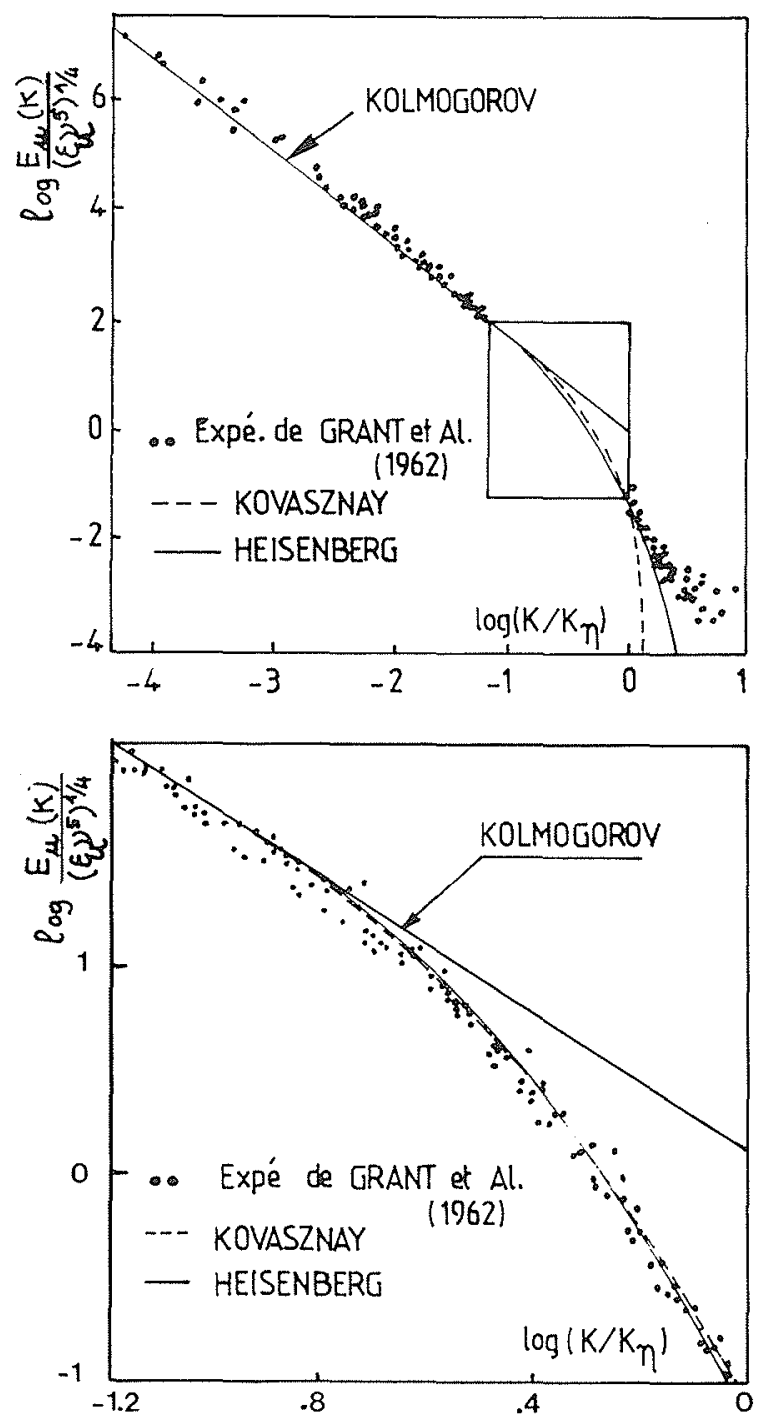

Figures 12 et 13 - Spectre de l'énergie cinétique turbulente Comparaison expérience-théories.
Pao (1965) ainsi que Obukhov et Yaglom (1953) puis Novikov $(1961)^{(6)}$.

'On trouve dans le livre de Panchev (1971), p. 244) les figures 12 et 13 qui illustrent la comparaison entre les expériences de Grand et al. (1962) et les prédictions théoriques de Kovasznay (1948) et Heisenberg (1948). Nous constatons que l'analyse de Kolmogorov est en défaut dès que $K \geq 0,1 K_{n}$.

On voit que l'on ne peut prétendre, compte tenu de la dispersion des points expérimentaux, que l'une des théories est meilleure que l'autre ${ }^{(7)}$. Néanmoins, la théorie d'Heisenberg est plus fidèle à l'expérience sur une gamme plus étendue de nombres d'ondes. C'est pourquoi on l'utilise fréquemment.

On se sert aussi de l'analyse de Pao (1965) en raison de la commodité de son expression analytique qui se prête bien aux ajustements de courbes sur les points expérimentaux.

Diverses expressions analytiques, proposées par les auteurs ci-dessus mentionnés, sont consignées dans le tableau I. $\widetilde{K}$ est le nombre d'onde réduit $K / K_{\eta}$ où $K_{\eta}$ est égal à $\left(\left\langle\epsilon_{u}>\nu^{-3}\right)^{1 / 4}\right.$.

Pour $K \ll K_{\eta}$ les spectres tendent vers la loi en puissance $-5 / 3$ sous la forme déjà indiquée plus haut, c'est-à-dire :

$$
E_{u}(K)=C<\epsilon_{u}>^{2 / 3} K^{-5 / 3}
$$

La constance $C$ a fait l'objet de nombreuses études expérimentales dans diverses conditions ou types d'écoulements : canal [Comte-Bellot (1965)], jet plan [Bradbury (1965)], jet axisymétrique [Gibson et al. (1971)], sillages [Uberoi et Freymuth $(1969,1970)]$.

(6) Ces deux dernières références peuvent être trouvées dans le livre de Tatarskii (1971).

(7) Une étude comparée des spectres d'énergie peut être trouvée dans l'article de Ellison (1962). 
Les valeurs trouvées sont toutes proches de 1,5. On trouvera dans Monin et Yaglom (1975, T2, p.-483) une bibliographie importante concernant les diverses mesures de la constante de Kolmogorov ainsi qu'une étude critique des conditions dans lesquelles elles ont été effectuées, valeur du nombre de Reynolds notamment.

\section{Résultats concernant le champ de pression}

\subsection{Zone d'équilibre}

La première hypothèse de Kolmogorov s'applique au champ de la pression turbulente.

La fonction de structure:

$$
\left\langle\Delta p^{2}(r)\right\rangle=\langle[\partial p(\vec{x}+\vec{r}, t)-\partial p(\vec{x}, r)]\rangle
$$

s'écrit dans la zone d'équilibre :

$$
<\Delta p^{2}(r)>=\rho^{2}<\epsilon_{\mathrm{u}}>g\left(\frac{r}{\eta}\right)
$$

où $g$ est une fonction universelle de la variable réduite $(r / \eta)$

Dans le domaine inertiel, (2) ne doit pas contenir la viscosité cinématique, de sorte que l'on a nécessairement :

$$
<\Delta p^{2}(r)>=\rho^{2}<\epsilon_{u}>^{4 / 3} r^{4 / 3}
$$

Le spectre correspondant à (3) s'obtient par transformée de Fourier et donne :

$$
E_{p}(K)=C^{2}<\epsilon_{u}>^{4 / 3} K^{-7 / 3}
$$

où $C$ est la constante de Kolmogorov [8(1)].

\subsection{Analyse de Batchelor}

Elle s'appuie sur l'hypothèse de quasi-normalité des fluctuations de la vitesse locale.

Partant des équations de Navier et de l'équation de continuité, Batchelor (1951) explicite le champ de la pression fluctuante sous la forme :

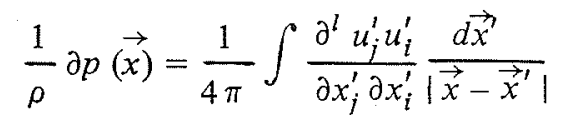

où $u_{i}^{\prime}$ est la composante de la vitesse au point $\vec{x}^{\prime}$.

Le calcul de la fonction de corrélation de $\partial p$ fait intervenir le moment du quatrième ordre du champ de vitesse qui s'exprime simplement en fonction des moments d'ordre 2 lorsque l'on a affaire à une loi de Gauss. On aura, par exemple :

$$
\begin{aligned}
& <u_{i} u_{j} u_{l}^{\prime} u_{m}^{\prime}>=<u_{i} u_{j}><u_{l}^{\prime} u_{m}^{\prime}>+ \\
& +\quad<u_{i} u_{l}^{\prime}><u_{j} u_{m}^{\prime}>+<u_{i} u_{m}^{\prime}><u_{j} u_{l}^{\prime}>
\end{aligned}
$$

Batchelor calcule la quantité :

$$
P \overrightarrow{(r)}=\frac{1}{\rho^{2}}<\delta p(\vec{x}) \delta p(\vec{x}+\vec{r})>
$$

ainsi que la transformée de Fourier :

$$
\Pi(\vec{K})=\frac{1}{(2 \pi)^{3}} \int P(\vec{r}) e^{-i K \cdot \vec{r}} \overrightarrow{d r}
$$

avec :

$$
\Pi(\vec{K})=\frac{1}{\rho^{2}} \cdot E_{p p}(\vec{K})
$$

$E_{p p}(\vec{K})$ est la densité spectrale des fluctuations de pression.

La variance $\left\langle\delta p^{2}\right\rangle$ est donnée par :

$$
<\delta p^{2}>=4 \pi \rho^{2} \int_{0}^{\infty} K^{2} \Pi(K) d K
$$

d'où le spectre de la pression $E_{p}(K)$ :

$$
E_{p}(K)=4 \pi \rho^{2} K^{2} \Pi(K)
$$

Le résultat de Batchelor (1951) s'exprime sous la forme :

$\Pi(K)=\frac{1}{8 \pi^{2}} \int E_{u}\left(K^{\prime}\right) E_{u}\left(\left|\vec{K}-\vec{K}^{\prime}\right|\right) \frac{\sin ^{4} \varphi}{\left|\vec{K}-\vec{K}^{\prime}\right|^{4}} d \vec{K}^{\prime}$

$E_{u}(K)$ est le spectre d'énergie đonné par 8 (1) dans le cas $K \ll K_{\eta}$ ou plus généralement par le tableau I. $\varphi$ est l'angle $\left(\vec{K}, \vec{K}^{\prime}\right)$.

L'évaluation de l'intégrale de Batchelor présente une certaine difficulté. Néanmoins, un calcul numérique de (12) a été mené, dans le cas du spectre de Pao et du spectre d'Heisenberg, afin de connaître le comportement de la pression au voisinage de $K=K_{\eta}$.

Les résultats présentés ici concernent le spectre adimensionné :

$$
\widetilde{\Pi}(\widetilde{K})=\frac{\Pi(\tilde{K})}{\nu^{4} K_{\eta}} \quad \widetilde{K}=\frac{K}{K_{\eta}}
$$

Comme le spectre de l'énergie cinétique d'agitation turbulente, le spectre de pression décroit très rapidement au voisinage de $K=K_{\eta}$. La figure 14 montre la compa-

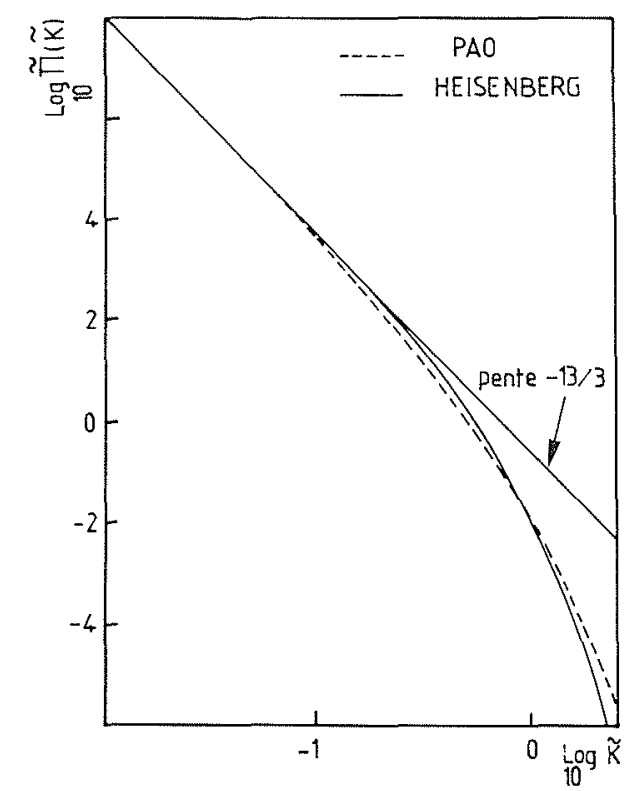

Figure 14 - Spectre de la pression calculé à partir du spectre d'énergie cinétique. 


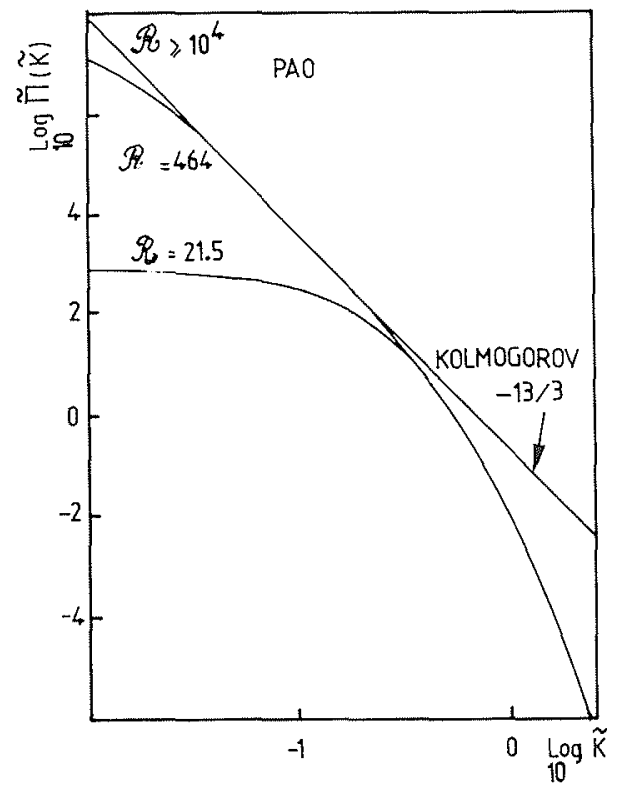

Figure 15 - Spectre de la pression selon la valeur du nombre de Reynolds de la turbulence.

raison entre un spectre de $\mathrm{Pao}$ et un spectre d'Heisenberg. (13) peut s'écrire sous la forme:

$$
\widetilde{\Pi}(\widetilde{K})=\frac{1}{64 \pi} C^{2} \tilde{K}^{-13 / 3} \widetilde{I}(\tilde{K}, R)
$$
$\sim \sim$

où $I(K, R)$ est la fonction de coupure du spectre de pression. On peut montrer, par le calcul, que $\widetilde{I}$ est quasiment indépendante du nombre de Reynolds $R$ de la turbulence ainsi qu'il est in diqué sur la figure 15 .

Dans le domaine inertiel, la densité spectrale de la pression varie comme la puissance $-13 / 3$ du nombre d'ondes, le spectre $E_{p}(K)$ correspondant étant donné par (4).

\section{Résultats concernant la température}

Le problème du transfert de chaleur en écoulement turbulent a été abordé pour la première fois par Obukhov (1949) et Corrsin (1951).

La température est considérée comme un scalaire inerte et passif. On veut dire par là qu'elle est transportée par le mouvement sans modification de celui-ci.

Le mouvement turbulent effectue un brassage du champ. Les gradients de température sont "mélangés", de sorte qu'il y a réduction des transferts thermiques et, par suite, homogénéisation de la température.

La théorie du mélange turbulent, voir par exemple : Monin et Yaglom (1975, T2, p. 377) est fondée sur l'équation de conduction:

$$
\frac{\partial}{\partial t}(\delta T)=\vec{u} \cdot \vec{\nabla}(\delta T)=\chi \nabla^{2}(\delta T)
$$

où $\delta T$ est la fluctuation de la température absolue notée $T, \vec{u}$ la partie fluctuante de la vitesse du fluide et $\chi$ la diffusivité thermique.
On trouve une équation de conservation analogue à 2 (43) soit :

$$
\frac{\partial}{\partial t}\left(\frac{1}{2}<\delta T^{2}>\right)=-<\epsilon_{T}>
$$

$\left\langle\epsilon_{T}\right\rangle$ est la taux moyen de dissipation de la variance $\left\langle\delta T^{2}\right\rangle$ des fluctuations de température qui est relié au champ $\delta T(\vec{x})$ par :

$$
<\epsilon_{T}>=\chi<\left[\frac{\partial(\delta T)}{\partial x_{i}}\right]^{2}>
$$

qui s'identifie formellement au taux moyen de dissipation de l'énergie cinétique turbulente, noté plus haut $\left\langle\epsilon_{u}>\right.$, et donne par 2 (54) et 2 (49).

On se limitera, à titre d'exemple, au cas des gaz pour lesquels le nombre de Prandtl $\mathscr{P}=v \chi^{-1}$ est de l'ordre de l'unité.

\subsection{Zone d'équilibre}

On applique la première hypothèse de Kolmogorov au champ des fluctuations de température en considérant la fonction de structure :

$$
\left.<\Delta T^{2} \overrightarrow{(r)}\right\rangle=\left\langle[\delta T(\vec{x}+\vec{r}, t)-\delta T \overrightarrow{(x, t)}]^{2}\right\rangle
$$

qui s'écrit dans la zone d'équilibre :

$$
\left.<\Delta T^{2} \overrightarrow{(r)}\right\rangle=\left\langle\epsilon_{T}><\epsilon_{u}>^{-1 / 2} \chi^{1 / 2} h\left(r / \eta_{T}\right)\right.
$$

où $h$ est une fonction universelle de la variable adimensionnée $r / \eta_{T}$, avec :

$$
\eta_{T}=\chi^{3 / 4}<\epsilon_{u}>^{-1 / 4}
$$

$\eta_{T}$ est la taille du plus petit spot de température existant au sein de l'écoulement. Il est à comparer à la taille du tourbillon dissipatif $\eta$ donné par 6 (3). Dans le cas $\mathscr{P} \sim 1$, on voit que :

$$
\eta_{T} \approx \eta
$$

La température correspondante $T_{\eta}$ est donnée par :

$$
T_{\eta}=\chi^{1 / 4}<\epsilon_{u}>^{-1 / 4}<\epsilon_{T}>^{1 / 2}
$$

qui est l'analogie de la vitesse $v_{\eta}(\S 3.1$.).

Dans la zone dite inertielle et convective, la fonction de structure ne doit pas dépendre de $\chi$. De sorte que (5) doit nécessairement s'écrire :

$$
\Delta T^{2}(r)=\left\langle\epsilon_{T}><\epsilon_{u}>^{-1 / 3} r^{2 / 3}\right.
$$

à une constante près de l'ordre de l'unité.

Dans le domaine où la diffusivité thermique atténue les fluctuations de température, et pour des distances de corrélation très petites devant l'échelle dissipative $\eta$, on a :

$$
<\Delta T^{2}(r)>=\frac{1}{3} \chi^{-1}<\epsilon_{T}>r^{2}
$$

Le spectre $E_{T}(K)$ correspondant à (9) s'obtient par transformée de Fourier et l'on montre qu'il est de la forme :

$$
E_{T}(K)=\left\langle\epsilon_{T}><\epsilon_{u}>^{-1 / 3} K^{-5 / 3}\right.
$$




\subsection{Domaine des grands nombres d'ondes}

Lorsque $K$ devient voisin de $K_{\eta}$, (11) ne s'applique plus. Il convient de prendre en compte l'amortissement visqueux et l'atténuation due à la diffusivité thermique.

Pour cela, Pao (1965) propose d'utiliser ses hypothèses sur le transfert dans un domaine étendu de nombres d'ondes $K \gtrsim K_{\eta}$. Il obtient pour

$$
\begin{aligned}
E_{T}(K)=\alpha & <\epsilon_{T}>. \\
& <\epsilon_{u}>^{-1 / 3} K^{-5 / 3} e^{-\frac{3}{2} \alpha x<\epsilon_{u}>^{-1 / 3} K^{4 / 3}}
\end{aligned}
$$

où $\alpha$ est une constante dont les valeurs expérimentales sont assez dispersées autour d'une valeurs moyenne voisine de 0.5, [Monin et Yaglom (1975, T2, p. 494)].

La densité spectrale $E_{T T}(K)$ correspondante sera :

$$
\begin{aligned}
E_{T T}(K) & =\frac{\alpha}{4 \pi}<\epsilon_{T}> \\
& <\epsilon_{u}>^{-1 / 3} K^{-11 / 3} e^{-\frac{3}{2} \alpha x<\epsilon_{u}>^{-1 / 3} K^{4 / 3}}
\end{aligned}
$$

soit, en normalisant les nombres d'ondes :

$$
\hat{E}_{T T}(\tilde{K})=\frac{\alpha}{4 \pi}<\epsilon_{T}>K_{\eta}^{-5} \nu^{-1} \tilde{K}^{-11 / 3} \tilde{J}(\tilde{K})
$$

avec :

$$
\widetilde{J}(\tilde{K})=e^{-\frac{3}{2} \alpha-1 \tilde{K}^{4 / 3}}
$$

$\widetilde{J}(\widetilde{K})$ est la fonction de coupure assurant une décroissance rapide du spectre de température au voisinage de $\widetilde{K}=1$.

Tatarskii (1971, p. 67) propose l'expression :

$E_{T T}(K)=0,033<\epsilon_{T}>\left\langle\epsilon_{u}\right\rangle^{-1 / 3} K^{-11 / 3} e^{-K^{2} / K_{m}^{2}}$

avec

$$
K_{m}=2,596 \mathscr{P}^{3 / 4} K_{\eta}
$$

soit, en normalisant les nombres d'ondes à $K_{\eta}$ :

$$
\begin{aligned}
E_{T T}(\tilde{K}) & =\frac{0,415}{4 \pi} . \\
< & \epsilon_{T}>K_{\eta}^{-5} \nu^{-1} \widetilde{K}^{-11 / 3} e^{-0,348 a^{-3 / 2} \widetilde{K}^{2}}
\end{aligned}
$$

La fonction de coupure correspondante s'écrivant :

$$
\widetilde{\sim} \widetilde{J}(K)=e^{-0,348 T^{-3 / 2} \widetilde{K}^{2}}
$$

Introduisons la densité spectrale adimensionnée $\widetilde{E}_{T T}(\widetilde{K})$ par :

$$
\tilde{E}_{T T}(\tilde{K})=\frac{\hat{E}_{T T}(\tilde{K})}{\chi^{-1}<\epsilon_{T}>K_{\eta}^{-5}}
$$

Pour Pao, on aura :

$$
\tilde{E}_{T T}(\tilde{K})=\frac{\alpha}{4 \pi} \mathscr{P}^{-1} \tilde{K}^{-11 / 3} e^{-\frac{3}{2} \alpha T^{-1} \widetilde{K}^{4 / 3}}
$$

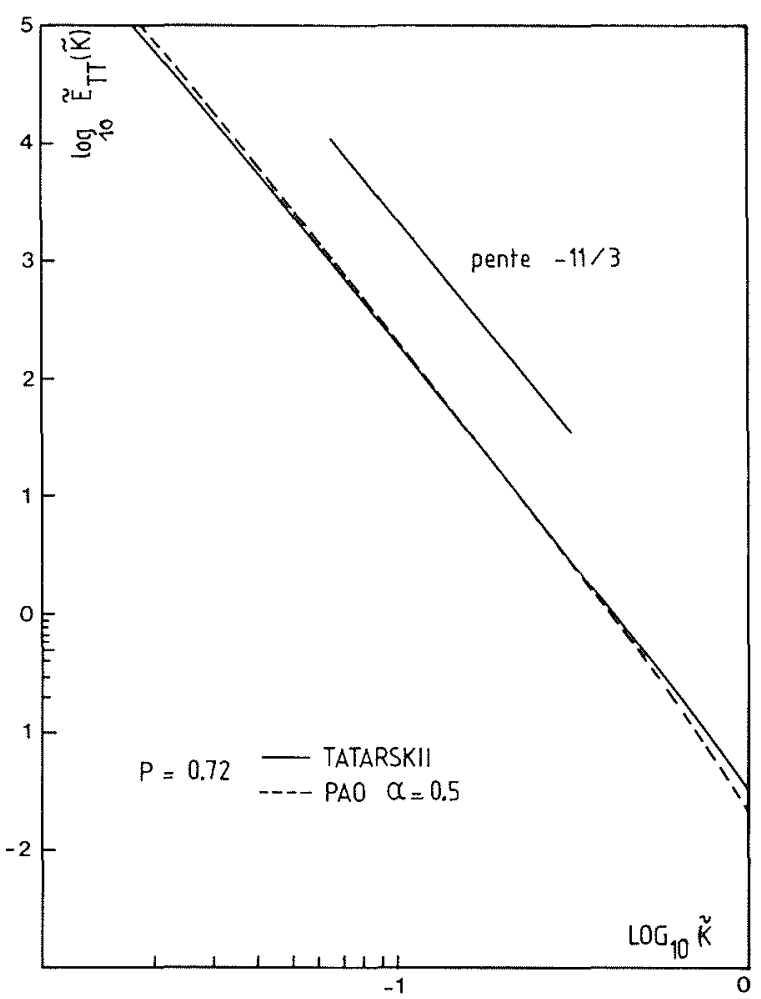

Figure 16 - Spectre de la température.

et pour Tatarskii :

$$
\tilde{E}_{T T}(\tilde{K})=\frac{0,415}{4 \pi} \mathscr{P}^{-1} \tilde{K}^{-11 / 3} e^{-0,148}-3 / \tilde{K}^{2}
$$

Sur la figure 16 , on a représenté les graphes de $E_{T T}(K)$ correspondant à (21) et (22).

\section{Bibliographie}

BATCHELOR G.K., TOWNSEND A.A. - The nature of turbulent motion at large wave-numbers. Proc. Roy. Soc., 1949, A 199, p. 238.

BATCHEIOR G.K. - Pressure fluctuations in isotropic turbulence. Proc. Cambridge Phil. Soc., 1951, 47, p. 359.

BATCHELOR G.K. - Small-scale variation of convected quantities like temperature in turbulent fluid. Part 1 : general discussion and the case of small conductivity. Joum. of Fluid mech., 1959, 5, 8, p. 113

BATCHELOR G.K. - An introduction to Fluid Dynamics. Cambridge U.P., 1967.

BATCHELOR G.K. - The theory of homogeneous turbulence. $6^{\mathrm{e}}$ ed. Cambridge U.P., 1970.

BATCHELOR G.K., TOWNSEND A.A. - Decay of turbulence in the final period. Proc. Roy. Soc., 1948, A, 194, p. 527

BELL T.L., NELKIN M. - Non linear cascade model for fully developped turbulence. Phys. Fluids, 1977, 20, p. 345.

BERGE P., POMEAU Y. - La turbulence. La Recherche, 1980, 11,110, p. 422.

BRADBURY L.J.S. - The structure of a self preserving turbulent plane jet. Joum. Fluid. Mech. 1965, 23, 1, p. 31.

CHANDRASEKHAR S. - On Heisenberg's elementary theory of turbulence. Proc. R. Soc., 1949, A. 200, p. 20.

CHANDRASEKHAR S. - The theory of turbulence, J. Madras Univ., 1957, 27, 1, p. 251. 
COMPTE-BELLOT G. - Ecoulement turbulent entre deux parois parallèles. P.S.T. Ministère de l'air $1965 n^{\circ} 419$, Paris. CORRSIN S. - On the spectrum of isotropic temperature fluctuations in an isotropic turbulence. Joum. of applied physics $1951,22,4$, p. 469.

CORRSIN S. - Outline of some Topics in homogeneous turbulent Flow. Journal of Geophysical Research, 1959, 64, 12, p. 2134.

CORRSIN S. - Turbulent Flow, American Scientist, 1961, 49 , p. 300.

CRAYA A. - Turbulence, Colloque $\mathrm{Cl}$ supp. du $\mathrm{n}^{\circ} 1$, Journ. Phys, 1976, 37, p. Cl-35.

ELLISON T.H. - The universal small scale spectrum of turbulence a high Reynolds number. Mécanique de la turbulence, p. 114, Colloque C.N.R.S., 1962, Marseille.

FAVRE A., KOVASZNAY L.S.G., DUMAS R., GAVIGLIO J., COANTIC M. - La turbulence en mécanique des fluides. Gauthiers-Villars, 1976, Paris.

FRISCH U., LESIEUR M., BRISSAUD A. - A Markovian random coupling model for turbulence. J. Fluid Mech., 1974,65, p. 145 .

FROST W., MOULDEN T.H. - Handbook of Turbulence, Vol. 1, Plenum Press, 1977, New-York.

GOLDSTEIN S. - On the law of decay of homogeneous isotropic turbulence and the theories of the equilibrium and similarity spectra Proc. Cam. Phil. Soc., 1951, 47, 3, p. 554.

GOLLUB J.P., SWINNEY H.L. - Onset of turbulence in a rotating fluid. Phys. Rev. Letters, 1975, 35, 14, p. 927.

GRANT H.L., STEWART R.W., MOILLIET A. - Turbulence spectra from a tidel channel, J. Fluid Mech., 1962, 12, 2 , p. 241 .

GURVICH A.S., ZUBKOVSKII S.L. - Estimate of the structure function of temperature fluctuation in the atmosphere. Izv. Akad. Nauk. SSR, Fig. Atmosf. i Okeana, 1966, 2, 2, p. 202.

GURVICH A.S., YAGLOM A.M. - Breakdown of Eddies and probability Distributions for small-scale of turbulence. The Phys. Fluids, 1967, supp. 10, 9, part II, p. 859.

HEISENBERG W. - Zur statistischen Theorie der Turbulenz, Z. Phys., 1948, 124, p. 628.

HEISENBERG W. - On the theory of statistical and isotropic turbulence, Proc. Roy. Soc, 1948, A, 195, p. 402.

HINZE J.O. - Turbulence, McGraw Hill, 1959, New York.

KARMAN T. - Progress in the statistical theory of turbulence, Proc. Nat. Acad. Sci, U.S.A., 1948, 34, 11, p. 530.

KOLMOGOROV A.N. - Local structure of turbulence in non compressible fluid with very high Reynolds number, Doklady Akad. Nauk. SSR, 1941, 30, 4, p. 299.

KOLMOGOROV A.N. - Dissipation of energy in locally isotropic turbulence, Doklady Akad. Nauk. SSR, 1941, 32, 1, p. 191

KOLMOGOROV A.N. - On the decay of isotropic turbulence in non compresible fluid. Doklady Akad. Nauk. SSR, 1941, 31,6, p. 147 .

KOLMOGOROV A.N. - Précisions sur la structure locale de la turbulence dans un fluide visqueux aux grands nombres de Reynolds, Mccanique de la turbulence, p. 29, Colloquc C.N.R.S., $n^{\circ} 108,1962$, Marseille.

KOLMOGOROV A.N. - A refinement of previous hypotheses concerning the local structure of turbulence in a viscous incompressible fluid at high Reynolds number. Jour. Fluid. Mech., 1962, 13, p. 82 .

KOVASZNAY L.S.G. - Spectrum of locally isotropic turbulence. J. Aeronaut. Sci. 1948, 15, 12, p. 745.

KRAICHNAN R.H. - On Kolmogorov's inertial-range theories. Journ. Fluid. Mech., 1974, 62, p. 305.

LANDAU L. - On the problem of turbulence. In : Collected Papers of L.D. Landau, 1944, p. 387. Ed. D ter Haar, Pergamon Press, Oxford, 1965.

LANDAU L., LIFCHITZ E. - Mécanique des fluides, notamment le chapitre III : turbulence. Ed. Mir, 1971.

LEE D.A. - Statistical theory of turbulence. V.K.I. Lecture series 17,1970 .

LEITH C.E. - Diffusion approximation to inertial energy transfer in isotropic turbulence Phys. of Fluids, 1967, 10,7, p. 1406 .
LESLIE D.C. - Review of developments in turbulence theory, Rep. Prog. Phys., 1973, 36, p. 1365.

LIN C.C. - Statistical Theories of turbulence. High speed Aerodynamics and jet Propulsion, 1959, Vol. V, p. 197, Princeton U.P.

LIN C.C., REID W.H. - Turbulent Flow - Theoretical aspects Hand. Phys., 1963, 8, 2, p. 438.

MANDELBROT B.B. - Intermittent turbulence in self-similar cascades. Jour. Fluid. Mech., 1974, 62, p. 331.

MANDELBROT B.B. - Intermittent turbulence and fractal dimension in Turbulence and Navier Stokes dimension. $\mathrm{R}$. Teman ed. Lecture notes in Mathematics, 1976, p. 121. Springer-Verlag.

MARTIN P.C. - Instabilités, oscillations et chaos. Journ. Phys., 1976, 37, p. Cl-57.

MONIN A.S., YAGLOM A.M. - Statistical Fluid Mechanics, Vol 1 et 2, The Mit Press Cambridge.

NELKIN M. - Turbulence, critical fluctuations and intermittency, Phys. Rev., 1974, A9, 1, p. 388.

NELKIN M. - Pl,ysique statistique de la turbulence. Collège de France, 1976, Paris.

NIHOUL J.C.J. - The statistical theory of Turbulence, V.K.I. Lecture series $17,1970$.

NOVIKOV E.A. - The energy spectrum of incompressible turbulent flow, Dokl. Akad. Nauk. SSR, 1961, 139, 2, p. 331 .

NOVIKOV E.A. - Change of energy dissipation in a turbulent flow and the spectral distribution of energy. P.M.M., 1963, 17,5, p. 944 .

NOVIKOV E.A. - Similarity of scale for stochastic fields, Dokl. Akad. Nauk. SSR, 1969, 184, 4, p. 104.

NOVIKOV E.A. - Intermittence et similitude d'échelle dans la structure d'un écoulement turbulent. Prikl. Mat. Mekh., 1971, 35, p. 266. (Appl. Math. Mech. 35, p. 231).

OBUKHOV A.M. - On the distribution of energy in the spectrum of turbulent flow. Dokl. Akad. Nauk. SSR, 1941 $30,22$.

OBUKHOV A.M. - Statistically homogeneous field on sphere. USPEHY Math. Sci., 1947, 2, 2.

OBUKHOV A.M. - Structure of the temperature field in a turbulent flow. Izv. Akad. Nauk. SSR Geogr. i. Geofiz., $1949,13,1$, p. 58.

OBUKHOV A.M., YAGLOM A.M. - Microstructure of turbulent flow, 1951, 15, 1, p. 3, Traduction NACA TM 1350, 1953.

OBUKHOV A.M. - Probability description of continuous field, Trudy Geofiz in-Ta Akad. Nauk. SSR, 1954, 24, 151, p. 3.

OBUKHOV A.M. - Some specific features of atmospheric turbulence, J. Fluid. Mech., 1962, 13, p. 77.

ONSAGER L. - The distribution of energy in turbulence (résumé). Phys. Rev., 1945, 68, p. 286.

ONSAGER L. - Statistical hydrodynamics, Nuovo Cimento, 1949,6 supp. série 9, 2, p. 279.

ORSZAG S.A. - Lectures on the statistical theory of turbulence. 1977 , p. 237 , Ecole d'été des Houches 1973 . Fluid Dynamics Gordon and Breach.

PANCIIEV S. - Random Functions and Turbulence. Pergamon Press Oxford, 1971.

PAO Y.H. - Structure of turbulent velocity and scalar fields at large wave numbers. Phys. of Fluids, 1965, 8, 6, p. 1063.

PROUDMAN I. - A comparison of Heisenberg's spectrum of turbulence with experiment. Proc. Cambr. Phil. Soc., 1951, $47,1, p .158$.

RUELLE D., TAKENS F. - On the nature of turbulence. Comm. Math. Phys., 1971, 22, p. 167.

ROBERTSON H.P. - The invariant theory of isotropic turbulence. Proc. Cambr, Phil. Soc., 1940, 36, p. 209.

ROSE H.A., SULEM P.L. - Fully developped turbulence in statistical mechanics. Joum. Phys., 1978, 39, 5, p. 441.

SAFFMAN P.G. - The large-scale structure of homogeneous turbulence. $J$. Fluid Mech., 1967, 27, 3, p. 581.

TATARSKII V.I. - The effect of the turbulent atmosphere on wave propagation. Israel program for scientific translations. U.S. Department of Commerce NTIS, 1971

TENNEKES H., LUMLEY J.L. - A first course in turbulence, the Mit Press Cambride, 1972. 
UBEROI M.S., FREYMUTH P. - Spectra of turbulence in wakes behind circular cylinders. Phys. Fluids, 1969, 12, 7, p. 1359.

UBEROI M.S., FREYMUTH P. - Turbulent energy Balance and spectra of the axisymetric wake. Phys. Fluids, 1970, 13, 9, p. 2205.

VAN ATTA C.W., PARK J. - Statistical self similarity and inertial subrange turbulence. Lecture Notes in Physics $n^{\circ} 12$, Statistical models and turbulence, p. 402. Edité par M. Rosenblatt et C. Van Atta, Springer, Betlin, 1972.

WEIZSACKER C.F. - Das Spektrum der Turbulence bei grossen
Reynoldsschen Zahlen. Zeitschrift fur Physik, 1948, 124, p. 614 .

WYNGAARD J.C., PAO Y.J. - Some measurements on the fine structure of large Reynolds number turbulence. Lecture Notes in Physics, $n^{\circ} 12$ Statistical Models and Turbulence, p. 384, Edité par M. Rosenblatt et C. Van Atta, Springer, Berlin, 1972.

YAGLOM A.M. - Effect of fluctuations in energy dissipation rate on the form of turbulence characteristics in the inertial subrange. Dokl. Akad. Nauk. SSR, 1966, 166, 1, p. 49. Traduction dans Soviet Physics, 11, p. 26, 1966. 